\title{
Study of Possible Local Quasars and Search for a Quasar-Stellar Connec- tion. III. A Sample of 341 QSOs
}

\author{
Kiril P. Panov*
}

Institute of Astronomy and National Astronomical Observatory, Bulgarian Academy of Sciences, Sofia, Bulgaria

\begin{abstract}
A new sample of 341 possible local QSOs are presented, which are in the vicinity of low redshift galaxies. Physical characteristics of the sample quasars are determined and previously reported relations are confirmed: density - redshift, absolute mag - radius, absolute mag - mass, mass - radius, mass - luminosity, and mass - density. These relations seem to support the basic assumptions that quasars are single, compact objects, with dimensions close to their respective gravitational radius. Redshifts of quasars are dominated by a gravitational redshift component, and the gravitational redshifts seem to be quantized according to the Karlsson - sequence. Evidence is found in favour of the Arp's evolutionary scenario: QSOs are ejected from their respective parent galaxy and evolve as they recede, building new small mass companion galaxies. Evidence is found that in the course of evolution the quasar density and redshift decrease, while dimensions and luminosity increase. Relation luminosity - density is found in the sense that more dense quasars are less luminous.

Simple linear density equation is found, which seems to apply to quasars, but also to stars and may be even to planets, providing a possible link between these seemingly very different structures in the Universe. Evidence is found of possible increase of quasar mass and luminosity with increasing distance to about $\mathrm{z}=0.03$. The physics behind all these processes and relations remain unclear, but a revival of the old Ambartsumian's hypotheses seems possible, suggesting disintegration of an unknown primordial dense matter. The properties of quasars may invoke the need for deeper changes in our current theories. Most fascinating seems the possibility that a link may exist between quasars and stars.
\end{abstract}

Keywords: Active galaxies, Quasars, Gravitational redshifts, Evolution of quasars.

\section{INTRODUCTION}

The study of quasars is a most fascinating part of modern astrophysics and may have direct implications for our fundamental knowledge about the origin of galaxies. The standard theory about origin of galaxies is based on a gravitational collapse onto some previously built kernels. How these kernels were built in an expending Universe (even inflationary expending at the beginning) is still an open question. During the last decades, new ideas have been proposed, and which are radically deviating from conventional theories.

The origin of these unconventional ideas is the interpretation of quasar redshifts as non-cosmological. According to the Standard Quasar Model (SQM), most popular at present, the unprecedented large quasar redshifts are caused by the expansion of the Universe [1-3]. Assuming that quasars are at cosmological distances has an immediate consequence quasars have to be extremely luminous. According to the SQM quasars are huge black holes accreting matter [1-3]. Not only are the quasars' luminosities huge, but it also seems, judging by their visual magnitudes that with increasing cosmological distances quasar luminosity should increase $[4,5]$. On the face of it, such a conclusion could raise doubts. This problem needs, however, further consideration.

*Address correspondence to this author at the Institute of Astronomy and National Astronomcal Observatory, Bulgarian Academy of Sciences, Sofia, Bulgaria; Tel: 974 1910; Fax: 974 1910; E-mail: kpanov@astro.bas.bg
In the SQM, quasars are the most distant objects in the Universe, because of their large redshifts. There is a disturbing fact from the beginning, however - quasars do not follow the Hubble relation found for galaxies. How can we be sure we could apply the Hubble relation to determine the distances for quasars? A possible outcome of this problem may be the large spread of quasar luminosities. Although this may or may not be the real explanation of the problem, we should keep in mind the simple fact that quasars do not obey the Hubble relation, and a different cause for that may also exist.

Already during the first years after their discovery, attempts have been made to explain the quasar redshifts in different, "non-cosmological" ways. Among the most debated are the ideas based on the "intrinsic" origin of the redshifs: gravitational reddening [6-8], and the "variable mass hypothesis" $[9,10]$. The debate between the SQM and the "intrinsic-origin" hypothesis continues for more than 40 years, to this present day. If the redshifts of quasars are caused by intrinsic origin, the quasars are probably of local origin - local quasars. Both the SQM and the "intrinsic" views have their observational support and weak points. Strong observational support for the SQM comes from the observations of quasars hosted by galaxies, where in a few cases the redshift of the "hosted" quasar and that of the "hosting" galaxy are identical $[11,12]$. However, one should be aware of the observing difficulties and the possibility of "contamination" by quasar light when the hosting galaxy is observed. Moreover, there is at least one reported case, 
where quasar of $\mathrm{z}=2.114$ was found very close to the nucleus of the galaxy NGC 7319 with $z=0.022$ [13]. Clearly, the above argument can not be used by either side of this controversy, before further research work will have been done. There are a number of questions that the SQM leaves unanswered, or at least, not satisfactorily answered. I already mentioned the seemingly increasing quasar luminosity with the cosmological distance. There is also the question, why is the number of observed QSOs with $z>3$ decreasing? The number of quasars at larger cosmological distances is expected to increase and there has to be a reason for not seeing them. There is an interesting problem that concerns all hypothesis proposed for quasars - the Karlsson sequence of quasars redshifts. This is a sequence of specific and preferred redshifts for QSOs: 0.06, 0.30, 0.60, 0.96, 1.41, 1.96, and so on. The sequence could be obtained by: $\Delta \log (1+\mathrm{z})=0.089$ [14-17]. The Karlsson sequence was found with the early surveys of quasars but later not confirmed with modern redshift catalogues. On the face of it, this looks like a preliminary finding has been discarded by later, larger samples of data. For the SQM such an interpretation would be a relief. If confirmed, the Karlsson sequence would require (in the SQM) that the Universe should be expanding in shells of different and specific velocity values and that would be inconceivable. This sequence is a major obstacle also for the local origin hypothesis of quasars. In the framework of the gravitational reddening hypothesis an outcome could be found but only at a cost of a major sacrifice: a departure from a basic physical concept [18]. This will be discussed bellow. However, before we try to find an explanation of the Karlsson sequence, we have to answer the question, is the Karrlsson sequence real? If it is, why modern surveys could not confirm it?

Quasars release huge amount of energy. With the SQM, these energies have to be as large as $\sim 10^{45} \mathrm{ergs} / \mathrm{s}$ in a lifetime of $\sim 10^{7}-10^{8}$ years. In the framework of the gravitational reddening hypothesis, the energies released by local quasars have to be $\sim 10^{39}-10^{42} \mathrm{ergs} / \mathrm{s}$ [18]. How is this enormous energy output produced? In the SQM, the "engine" is provided by accretion onto a huge black hole. The local quasar model has as yet no specific physical engine. All we could say at this point is that the known physical processes are probably not sufficient to explain the quasar energy output.

The local quasar concept has also observational support. Since many years ago, researchers reported the association of high redshift QSOs with low redshift galaxies [19-30]. In some "discordant redshift associations", as Halton Arp calls them [19], there may be seen filaments, connecting the high redshift quasar with the low redshift galaxy. Prominent examples are NGC4319 and Mk205 [31], NGC3067 and 3 C232 $[32,33]$ etc. In other cases, quasars have been found very close to a low redshift galaxy $[13,34]$. Considerations show that a chance projection in these cases has a very low probability, $\sim 10^{-8}$ and lower $[35,36]$. Even large groups of quasars have been reported clustering around low redshift galaxies [37-41]. All these findings lead to the conclusion that quasars around low redshift active galaxies have been ejected by the respective parent galaxy [42-46].

Taking the same distance for a group of QSOs, as for their parent galaxy, it is possible to obtain some of the qua- sars' physical characteristics [18]. One more observational result should be mentioned, and which remains, in my opinion, a mystery for all proposed quasar models. If QSOs are the most distant objects in the Universe (according to SQM), they also have to be most young. One should expect that quasars are deficient in metals and a gradient of the metal abundances should exist in the sense that most distant quasars (largest redshifts) are most metal deficient. Surprisingly, high metal abundances were found in high redshift QSOs and no metal-gradient with distance so far has been claimed [47-49]. What do these findings mean, if confirmed? Are metals produced by rapidly evolved stellar populations around quasars, so soon after the Big Bang? Or, may be, yet another quasar riddle? This difficulty remains also for the gravitational reddening model, based on the disintegration of dense matter. Do we need to consider the possibility of an unknown process producing metals in a different way? The only way known to produce heavy elements at present are nuclear processes in stars, at late stages of their evolution. Especially interesting would be to look for a gradient of the metal abundances from higher to lower redshifts.

Some quasars exhibit jets of yet unknown nature (e.g. $3 \mathrm{C} 345$ in the vicinity of NGC6212). In some cases, moving structures were found by radio-observations along these jets $[50,51]$. If quasars are at cosmological distances, the velocities of these moving structures should be super-luminous. On the other hand, if quasars and their jets are of local origin (e.g. 3C345 would be at about the same distance as NGC6212), the "super-luminous" velocities will be reduced below the velocity of light. In the case of 3C 345 this velocity would be reduced to $0.33 \mathrm{c}$ [52].

Most decisive observations that can in principle distinguish between the "cosmological" and the "local" origin of quasars are observations of host galaxies.

During the last years, there are an increasing number of studies of host galaxies [53-58] which suggests that a solution of this problem in near future is possible.

In this paper, important astrophysical questions will be addressed and some tantalizing answers will be suggested. In the following I will use the procedure, outlined in $[18,59]$ to determine physical characteristics of a sample of 116 possible local quasars. These are added to the already published data [59] to build a sample of 341 possible local QSOs, and which are used to study relations for quasars. The sample of all 341 quasars studied is listed in Table $\mathbf{1 .}$

\section{DETERMINATION OF PHYSICAL CHARACTER- ISTICS OF LOCAL QUASARS.}

Assuming that the clustering of quasars around some active galaxies is real, as researchers claim, we could obtain some of the physical characteristics of QSOs [18, 59]. In these cases, it is possible to take for a group of quasars near an active galaxy the distance of the parent galaxy. The observed quasar redshift could be taken as composed by three components of different origin, according to Burbidge [60]:

$\left(1+z_{\mathrm{o}}\right)=\left(1+\mathrm{z}_{\mathrm{c}}\right) \cdot\left(1+\mathrm{z}_{\mathrm{gr}}\right) \cdot\left(1+\mathrm{z}_{\mathrm{d}}\right)$

In eq (1), $z_{0}$ is the observed redshift, $z_{c}$ is the cosmological redshift, $z_{g r}$ is the intrinsic redshift, specified here as gravitational redshift, and $z_{d}$ is the Doppler shift. As men- 
Table 1. Sample of 341 Local Quasars (Data from Veron-Cetty and Veron, 2010, 13 th ed.)

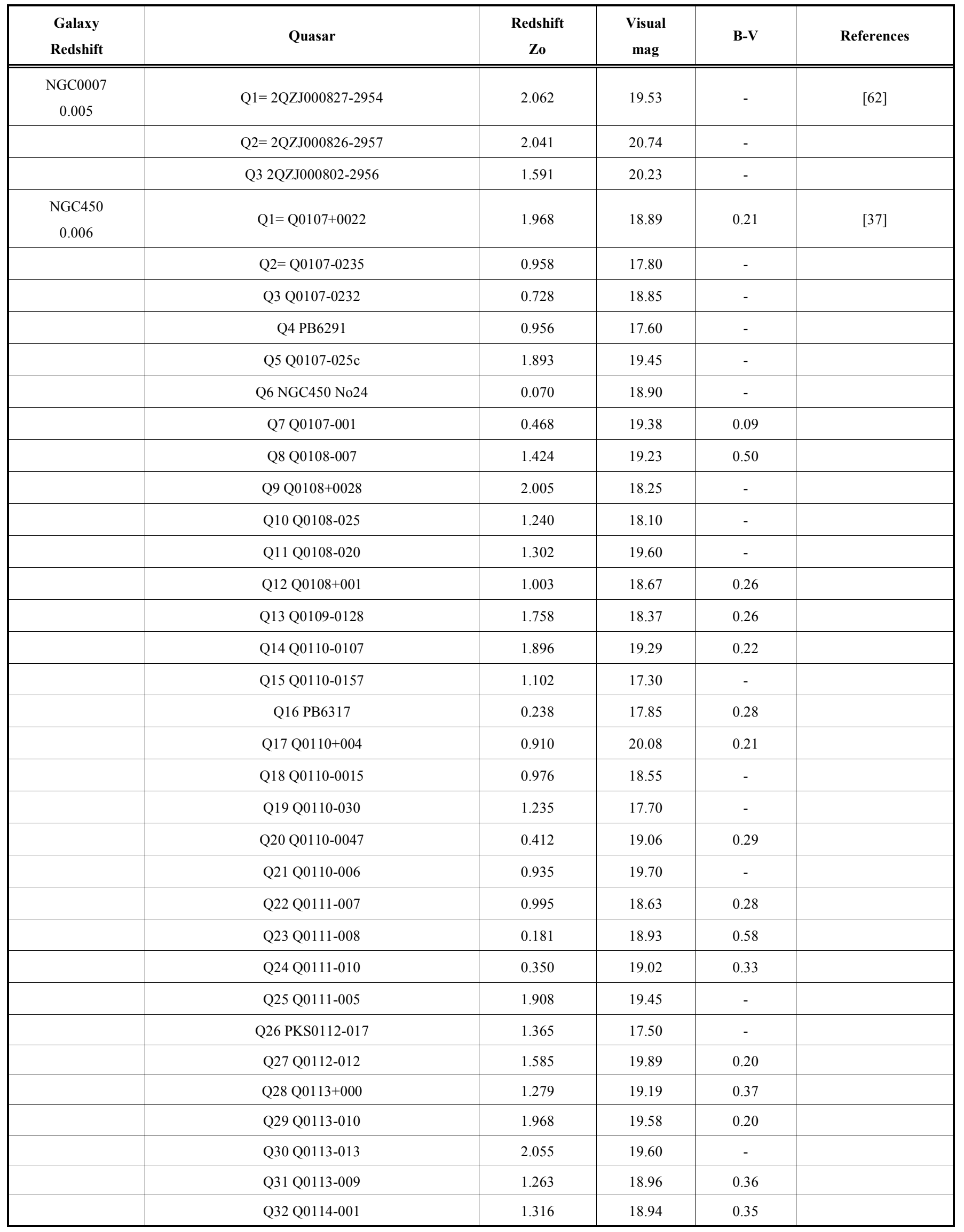


Table 1. contd...

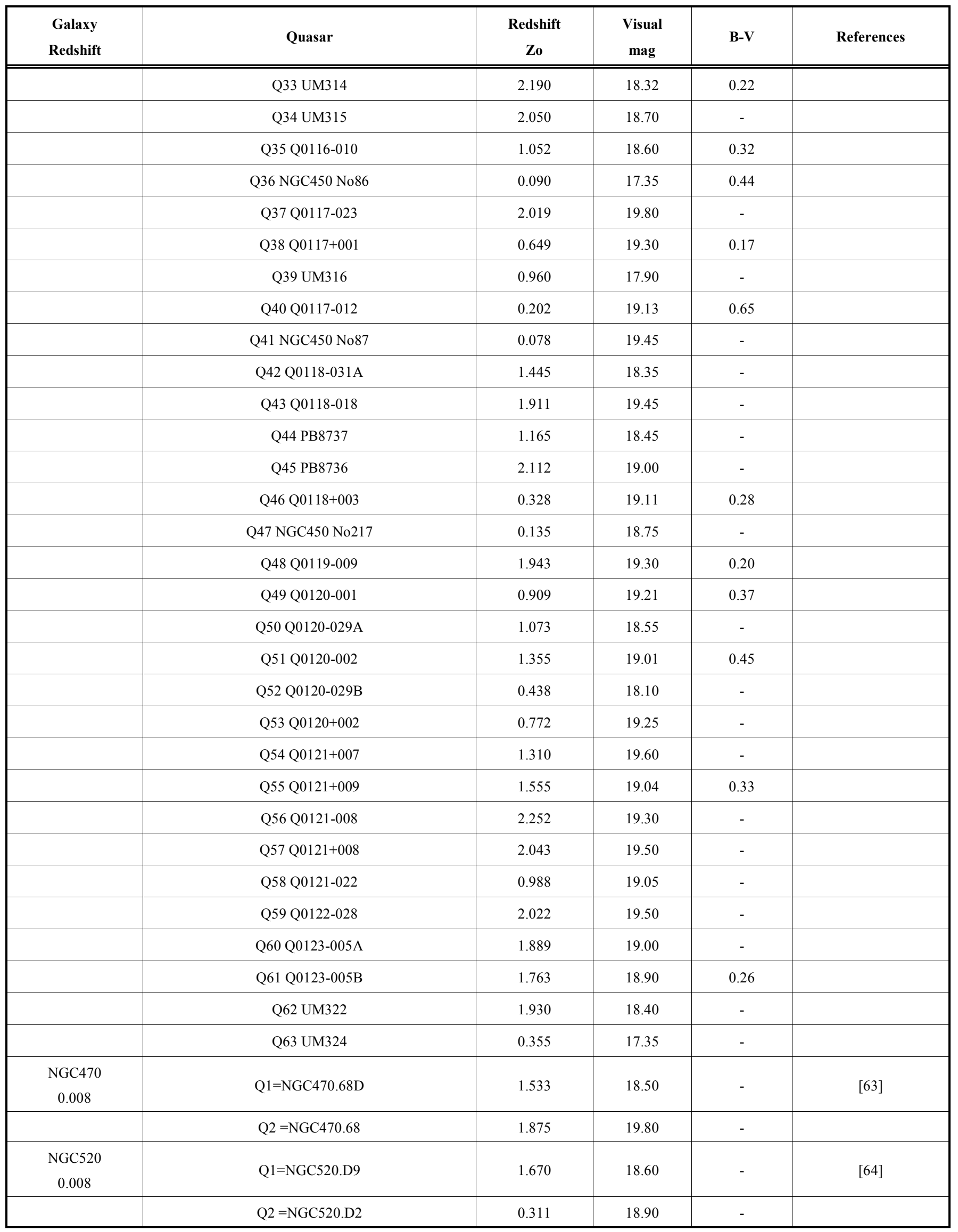


Table 1. contd...

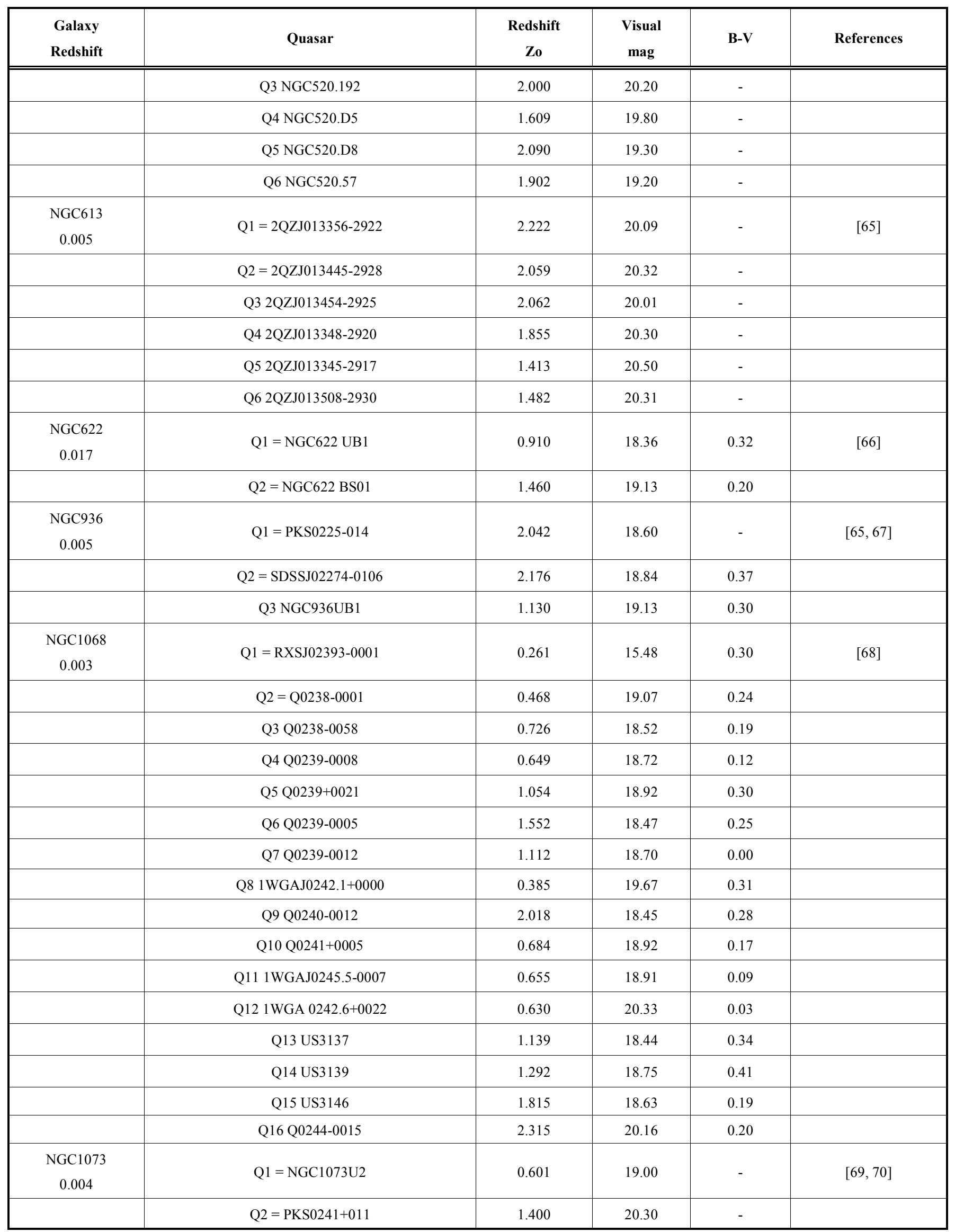


Table 1. contd...

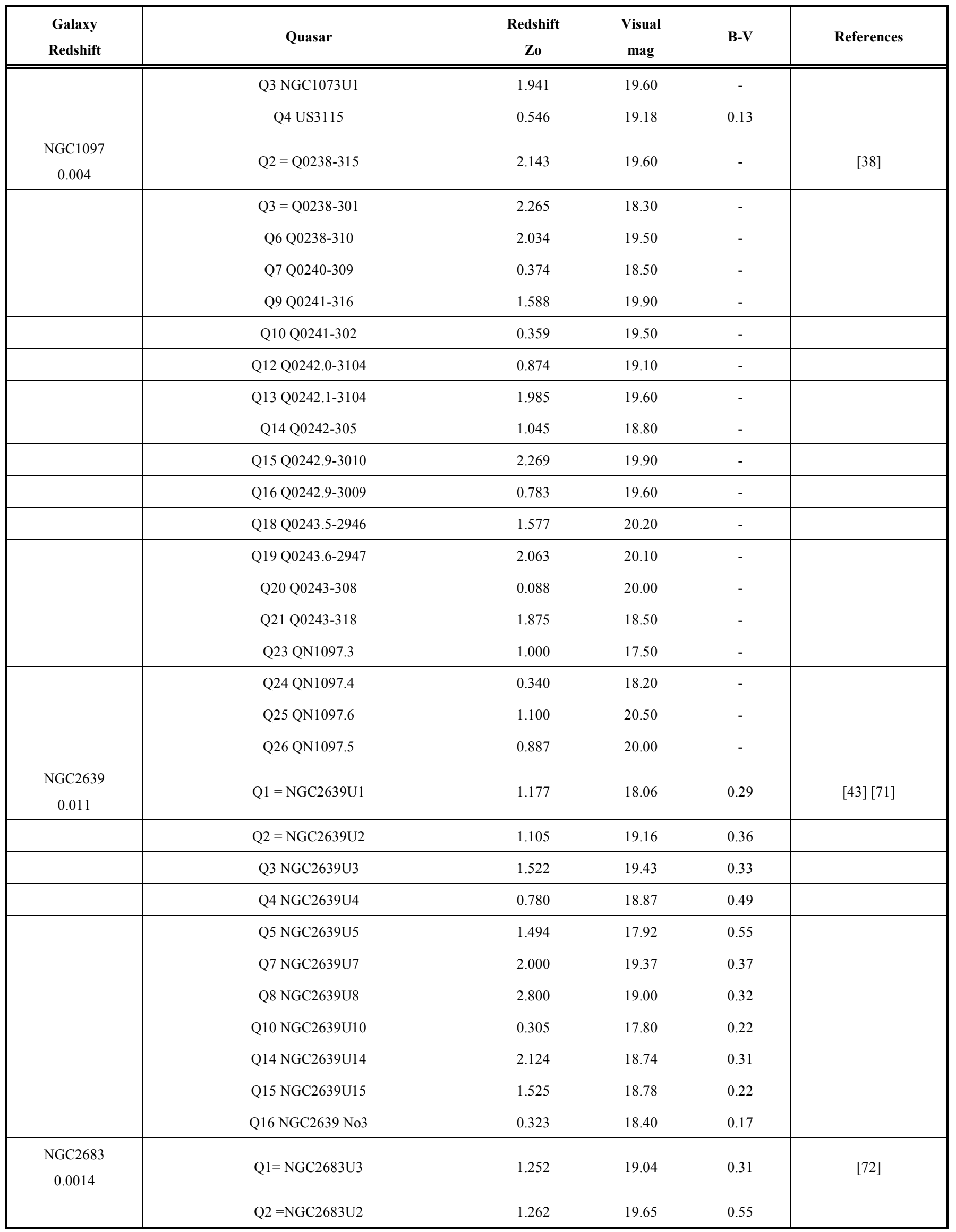


Table 1. contd...

\begin{tabular}{|c|c|c|c|c|c|}
\hline $\begin{array}{c}\text { Galaxy } \\
\text { Redshift }\end{array}$ & Quasar & $\begin{array}{l}\text { Redshift } \\
\text { Zo }\end{array}$ & $\begin{array}{c}\text { Visual } \\
\text { mag }\end{array}$ & B-V & References \\
\hline & Q3 NGC2683U8 & 0.065 & 18.60 & - & \\
\hline & Q4 NGC2683U1 & 0.621 & 17.79 & 0.15 & \\
\hline \multirow[t]{2}{*}{$\begin{array}{c}\text { NGC2841 } \\
0.0021\end{array}$} & $\mathrm{Q} 1=\mathrm{NGC} 2841 \mathrm{UB} 2$ & 0.120 & 18.70 & - & {$[73]$} \\
\hline & $\mathrm{Q} 2=\mathrm{NGC} 2841 \mathrm{UB} 1$ & 2.028 & 19.28 & 0.21 & \\
\hline \multirow[t]{4}{*}{$\begin{array}{c}\text { NGC2859 } \\
0.0056\end{array}$} & Q1= NGC2859U1 & 0.230 & 18.74 & 0.41 & {$[74]$} \\
\hline & Q2 =NGC2859U2 & 2.250 & 19.70 & - & \\
\hline & Q3 NGC2859U3 & 1.460 & 20.30 & - & \\
\hline & Q6 NGC2859U6 & 0.027 & 18.50 & - & \\
\hline \multirow[t]{5}{*}{$\begin{array}{c}\text { NGC2916 } \\
0.0124\end{array}$} & Q1= NGC2916UB5 & 1.546 & 19.23 & 0.35 & {$[73]$} \\
\hline & Q2 =NGC2916UB1 & 0.238 & 19.20 & - & \\
\hline & Q3 NGC2916UB2 & 0.793 & 19.00 & - & \\
\hline & Q4 NGC2916UB4 & 1.868 & 19.35 & 0.13 & \\
\hline & Q5 NGC2916UB3 & 1.279 & 19.09 & 0.43 & \\
\hline \multirow[t]{12}{*}{$\begin{array}{c}\text { NGC3034 } \\
0.001\end{array}$} & $\begin{array}{c}=\mathrm{M} 82 \\
\mathrm{Q} 1=\mathrm{M} 82 \text { No95 }\end{array}$ & 1.010 & 19.44 & 0.36 & {$[75]$} \\
\hline & Q2 = Hoag 1 & 2.048 & 19.50 & 0.30 & \\
\hline & Q3 Hoag 2 & 2.054 & 20.33 & 0.22 & \\
\hline & Q4 NGC3031U4 & 0.85 & 20.12 & 0.70 & \\
\hline & Q5 Hoag 3 & 2.040 & 20.31 & 0.16 & \\
\hline & Q6 Bol 105 & 2.240 & 21.40 & - & \\
\hline & Q7 M82 No69 & 0.930 & 19.38 & 0.70 & \\
\hline & Q8 M82 No22 & 0.960 & 19.04 & 1.31 & \\
\hline & Q9 Bol 75 & 0.740 & 22.00 & - & \\
\hline & Q10 Dahlem 7 & 0.675 & 19.80 & - & \\
\hline & Q11 Dahlem 12 & 0.626 & 18.90 & - & \\
\hline & Q12 Dahlem 17 & 1.086 & 17.99 & 0.33 & \\
\hline \multirow[t]{8}{*}{$\begin{array}{c}\text { NGC3079 } \\
0.004\end{array}$} & $\mathrm{Q} 1=\mathrm{SBS} 0953+556$ & 1.410 & 18.45 & 0.17 & {$[76]$} \\
\hline & $\mathrm{Q} 2=4 \mathrm{C} 55.17$ & 0.898 & 17.89 & 0.35 & \\
\hline & Q3 SBS0955+560 & 1.021 & 17.68 & 0.47 & \\
\hline & Q4 RXJ10005+5536 & 0.215 & 19.37 & 0.62 & \\
\hline & Q5 1WGAJ1000.9+5541 & 1.037 & 19.99 & 0.57 & \\
\hline & Q6 NGC3073UB1 & 1.530 & 19.04 & 0.32 & \\
\hline & Q7 ASV1 & 0.072 & 17.28 & - & \\
\hline & Q8 SBS0957+557 & 2.102 & 17.60 & - & \\
\hline
\end{tabular}


Table 1. contd..

\begin{tabular}{|c|c|c|c|c|c|}
\hline $\begin{array}{c}\text { Galaxy } \\
\text { Redshift }\end{array}$ & Quasar & $\begin{array}{c}\text { Redshift } \\
\text { Zo }\end{array}$ & $\begin{array}{c}\text { Visual } \\
\text { mag }\end{array}$ & B-V & References \\
\hline & Q9 Q0957+561A & 1.413 & 16.95 & 0.21 & \\
\hline & Q10 Q0957+561B & 1.415 & 16.95 & 0.21 & \\
\hline & Q11 ASV24 & 1.154 & 23.03 & - & \\
\hline & Q12 ASV31 & 0.352 & 21.14 & - & \\
\hline & Q13 MARK132 & 1.760 & 16.05 & 0.28 & \\
\hline & Q14 NGC3073UB4 & 1.154 & 18.38 & 0.38 & \\
\hline & Q15 1WGAJ1002.7+5558 & 0.219 & 21.20 & - & \\
\hline & Q16 Q0958+5625 & 3.216 & 20.08 & - & \\
\hline \multirow[t]{3}{*}{$\begin{array}{c}\mathrm{NGC} 3184 \\
0.002\end{array}$} & $\mathrm{Q} 1=\mathrm{NGC} 3184 \mathrm{UB} 4$ & 0.675 & 18.23 & 0.13 & [73] \\
\hline & $\mathrm{Q} 2=\mathrm{NGC} 3184 \mathrm{UB} 3$ & 0.920 & 19.21 & 0.35 & \\
\hline & Q3 NGC3184UB1 & 0.152 & 17.70 & - & \\
\hline \multirow[t]{8}{*}{$\begin{array}{c}\mathrm{NGC} 3384 \\
0.0023\end{array}$} & $\mathrm{Q} 1=\mathrm{NGC} 3384 \mathrm{U} 1$ & 0.442 & 19.31 & 0.19 & [77] \\
\hline & $\mathrm{Q} 2=\mathrm{NGC} 3384 \mathrm{U} 2$ & 1.280 & 19.27 & 0.34 & \\
\hline & Q4 NGC3384U4 & 1.107 & 19.06 & 0.25 & \\
\hline & Q5 NGC3384U5 & 1.192 & 20.00 & - & \\
\hline & Q8 NGC3384U8 & 1.134 & 18.56 & 0.45 & \\
\hline & Q13 NGC3384U13 & 0.497 & 19.57 & 0.43 & \\
\hline & Q14 NGC3384U14 & 0.520 & 19.94 & 0.21 & \\
\hline & Q15 NGC3384U15 & 1.131 & 19.76 & 0.44 & \\
\hline \multirow[t]{6}{*}{$\begin{array}{c}\text { NGC3516 } \\
0.009\end{array}$} & $\mathrm{Q} 1=1 \mathrm{WGAJ} 1107.7+7232$ & 2.100 & 18.50 & - & [78] \\
\hline & $\mathrm{Q} 2=1 \mathrm{WGAJ} 1105.4+7238$ & 1.399 & 20.00 & - & \\
\hline & Q3 1WGAJ1105.1+7242 & 0.930 & 20.00 & - & \\
\hline & Q4 1WGAJ1106.2+7244 & 0.690 & 19.10 & - & \\
\hline & Q5 1WGAJ1108.5+7226 & 0.328 & 20.20 & - & \\
\hline & Q6 NGC3516U2 & 1.710 & 18.60 & - & \\
\hline \multirow[t]{9}{*}{$\begin{array}{c}\text { NGC3628 } \\
0.003\end{array}$} & $\mathrm{Q} 1=$ Wee 47 & 1.413 & 19.06 & 0.26 & [39] \\
\hline & $\mathrm{Q} 2=$ Wee 48 & 2.060 & 18.91 & 0.26 & \\
\hline & Q3 Wee 50 & 1.750 & 19.58 & 0.18 & \\
\hline & Q4 Wee 51 & 2.150 & 19.44 & 0.29 & \\
\hline & Q8 Wee 52 & 2.430 & 20.97 & 0.24 & \\
\hline & Q9 Wee 55 & 1.940 & 19.06 & 0.26 & \\
\hline & Q10 Wee 36 & 2.490 & 20.70 & - & \\
\hline & Q11 Wee 38 & 2.370 & 20.05 & 0.48 & \\
\hline & Q12 Wee 45 & 2.100 & 20.12 & 0.08 & \\
\hline
\end{tabular}


Table 1. contd...

\begin{tabular}{|c|c|c|c|c|c|}
\hline $\begin{array}{l}\text { Galaxy } \\
\text { Redshift }\end{array}$ & Quasar & $\begin{array}{c}\text { Redshift } \\
\text { Zo }\end{array}$ & $\begin{array}{c}\text { Visual } \\
\text { mag }\end{array}$ & B-V & References \\
\hline & Q13 Wee 37 & 2.140 & 20.02 & 0.55 & \\
\hline & Q14 Wee 40 & 1.740 & 20.09 & 0.13 & \\
\hline & Q15 Wee 34 & 2.320 & 17.85 & 0.65 & \\
\hline & Q16 Wee 46 & 0.060 & 20.20 & - & \\
\hline & Q17 Wee 41 & 2.540 & 20.02 & 0.25 & \\
\hline & Q18 Wee 44 & 2.380 & 19.57 & 0.25 & \\
\hline & Q19 Wee 42 & 2.110 & 20.97 & 0.16 & \\
\hline & Q20 Wee 43 & 3.009 & 19.83 & 0.33 & \\
\hline \multirow[t]{3}{*}{$\begin{array}{c}\text { NGC3842 } \\
0.0211\end{array}$} & $\mathrm{Q} 1=\mathrm{Q} 1141+2013$ & 0.335 & 18.50 & - & {$[79,80]$} \\
\hline & $\mathrm{Q} 2=\mathrm{Q} 1141+2014$ & 0.946 & 19.08 & 0.24 & \\
\hline & Q3 Q1141+2012 & 2.200 & 20.18 & 0.25 & \\
\hline \multirow[t]{2}{*}{$\begin{array}{c}\text { NGC4235 } \\
0.007\end{array}$} & $\mathrm{Q} 1=\mathrm{PG} 1216+069$ & 0.334 & 15.65 & - & {$[71]$} \\
\hline & $\mathrm{Q} 2=1 \mathrm{ES} 1212+078(\mathrm{BL})$ & 0.137 & 16.00 & - & \\
\hline \multirow[t]{2}{*}{$\begin{array}{c}\text { NGC4258 } \\
0.002\end{array}$} & $\mathrm{Q} 1=\mathrm{QJ} 1218+472$ & 0.398 & 19.88 & 0.21 & {$[42]$} \\
\hline & $\mathrm{Q} 2=\mathrm{QJ} 1219+473$ & 0.654 & 19.43 & 0.17 & \\
\hline \multirow[t]{18}{*}{$\begin{array}{c}\text { NGC4410 } \\
0.025\end{array}$} & Q1 = SDSSJ12260+0853 & 2.237 & 19.57 & 0.27 & {$[40]$} \\
\hline & $\mathrm{Q} 2=\mathrm{SDSSJ} 12260+0912$ & 0.662 & 19.24 & 0.09 & \\
\hline & Q3 SDSSJ12255+0859 & 1.903 & 19.57 & 0.21 & \\
\hline & Q5 Q1222+0901 & 0.535 & 17.29 & 0.10 & \\
\hline & Q6 SDSSJ12273+0923 & 1.776 & 19.39 & 0.13 & \\
\hline & Q8 2E1225+0858 & 0.085 & 16.64 & 0.38 & \\
\hline & Q9 SDSSJ12281+0915 & 1.590 & 20.03 & 0.45 & \\
\hline & Q10 SDSSJ12279+0922 & 1.502 & 18.82 & 0.26 & \\
\hline & Q11 SDSSJ12261+0935 & 0.628 & 19.33 & 0.12 & \\
\hline & Q12 SDSSJ12238+0856 & 1.043 & 18.74 & 0.30 & \\
\hline & Q13 SDSSJ12235+0902 & 1.363 & 19.24 & 0.34 & \\
\hline & Q15 Q1225+0836 & 1.471 & 17.59 & 0.30 & \\
\hline & Q16 SDSSJ12178+0913 & 1.076 & 19.48 & 0.21 & \\
\hline & Q17 SDSSJ12240+0935 & 1.345 & 19.32 & 0.24 & \\
\hline & Q18 SDSSJ12230+0856 & 1.090 & 19.12 & 0.34 & \\
\hline & Q19 SDSSJ12231+0914 & 1.715 & 19.49 & 0.09 & \\
\hline & Q20 Q1220+0939 & 0.681 & 17.74 & 0.09 & \\
\hline & Q21 SDSSJ12291+0938 & 2.649 & 20.08 & 0.33 & \\
\hline
\end{tabular}


Table 1. contd...

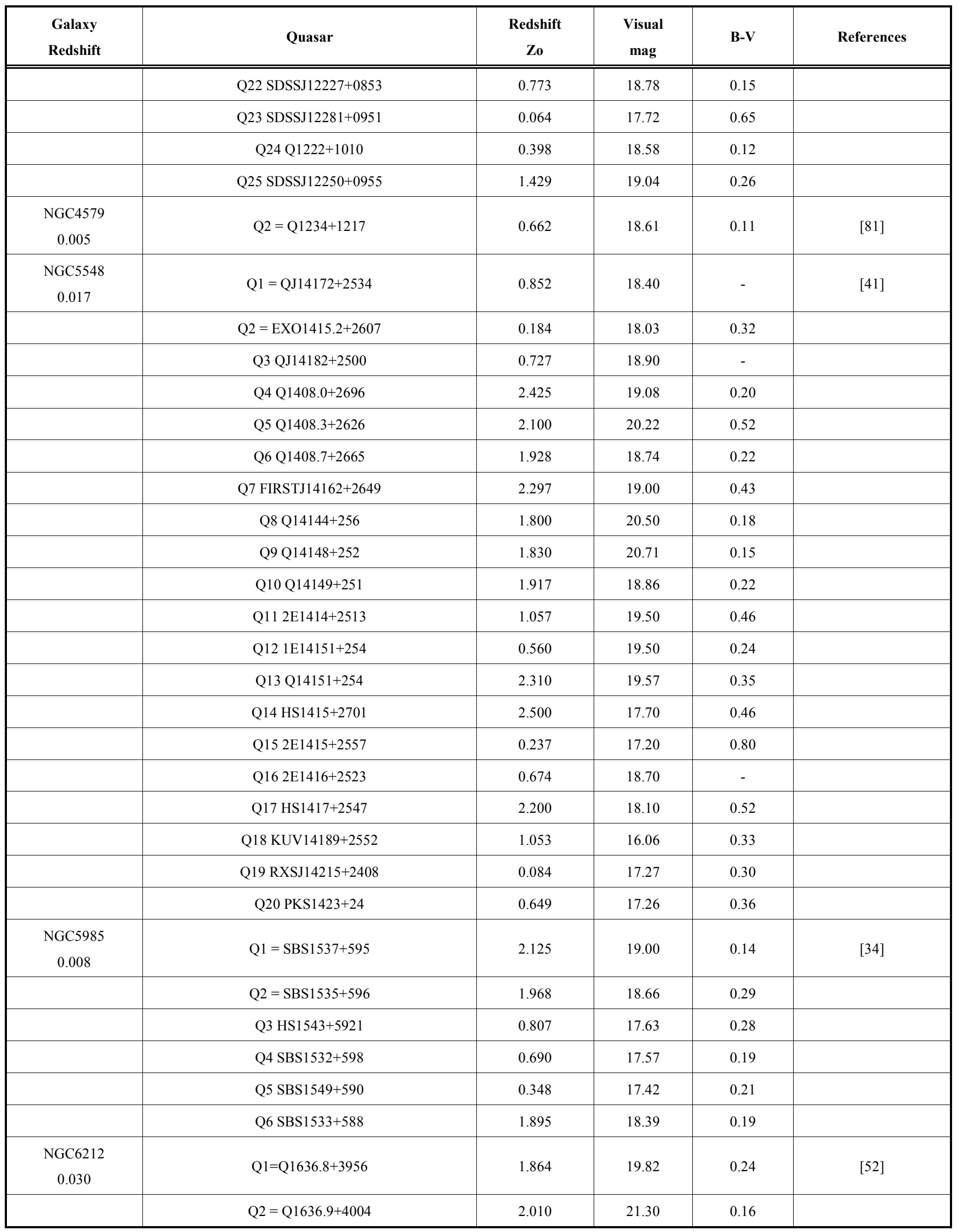


Table 1. contd....

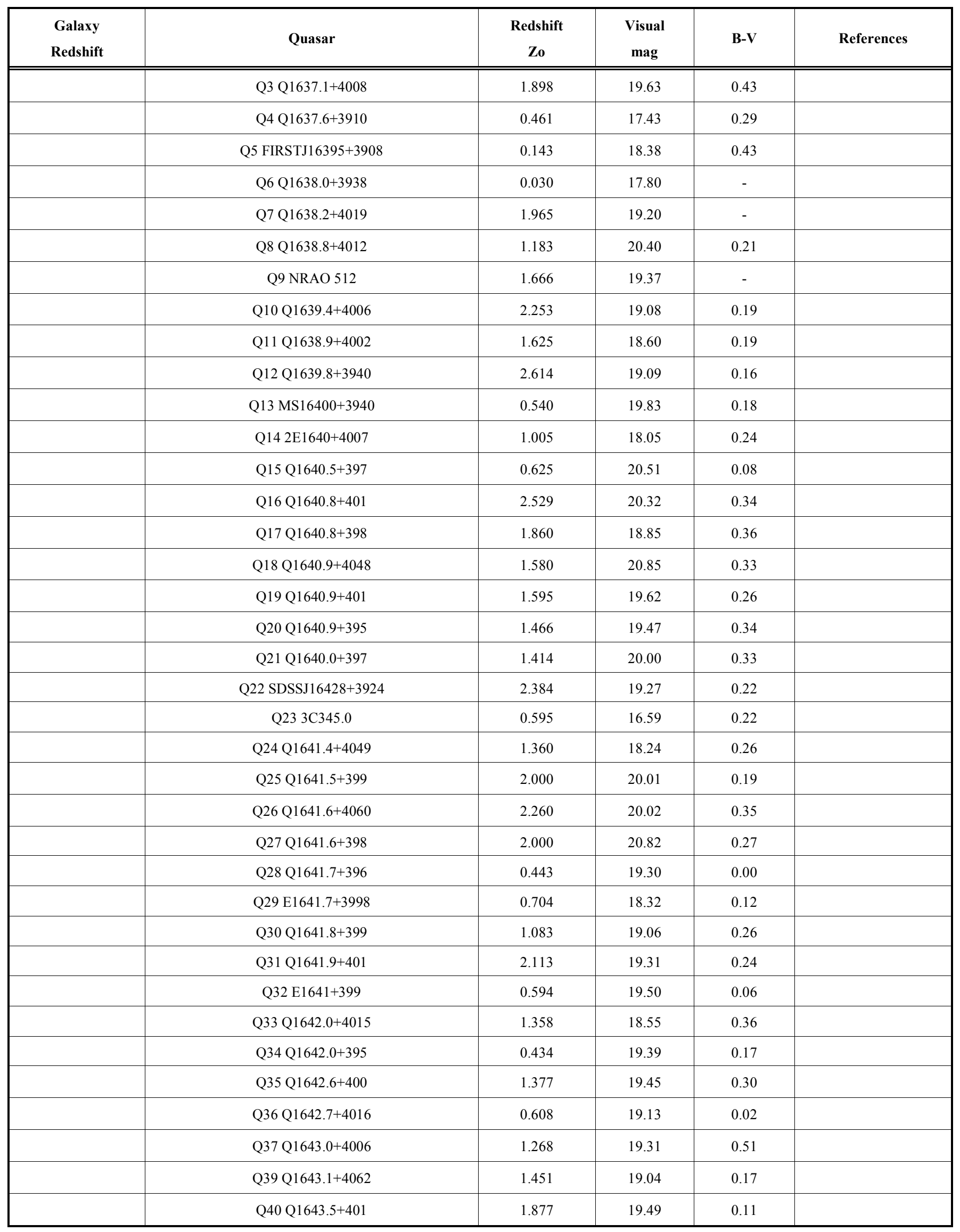


Table 1. contd....

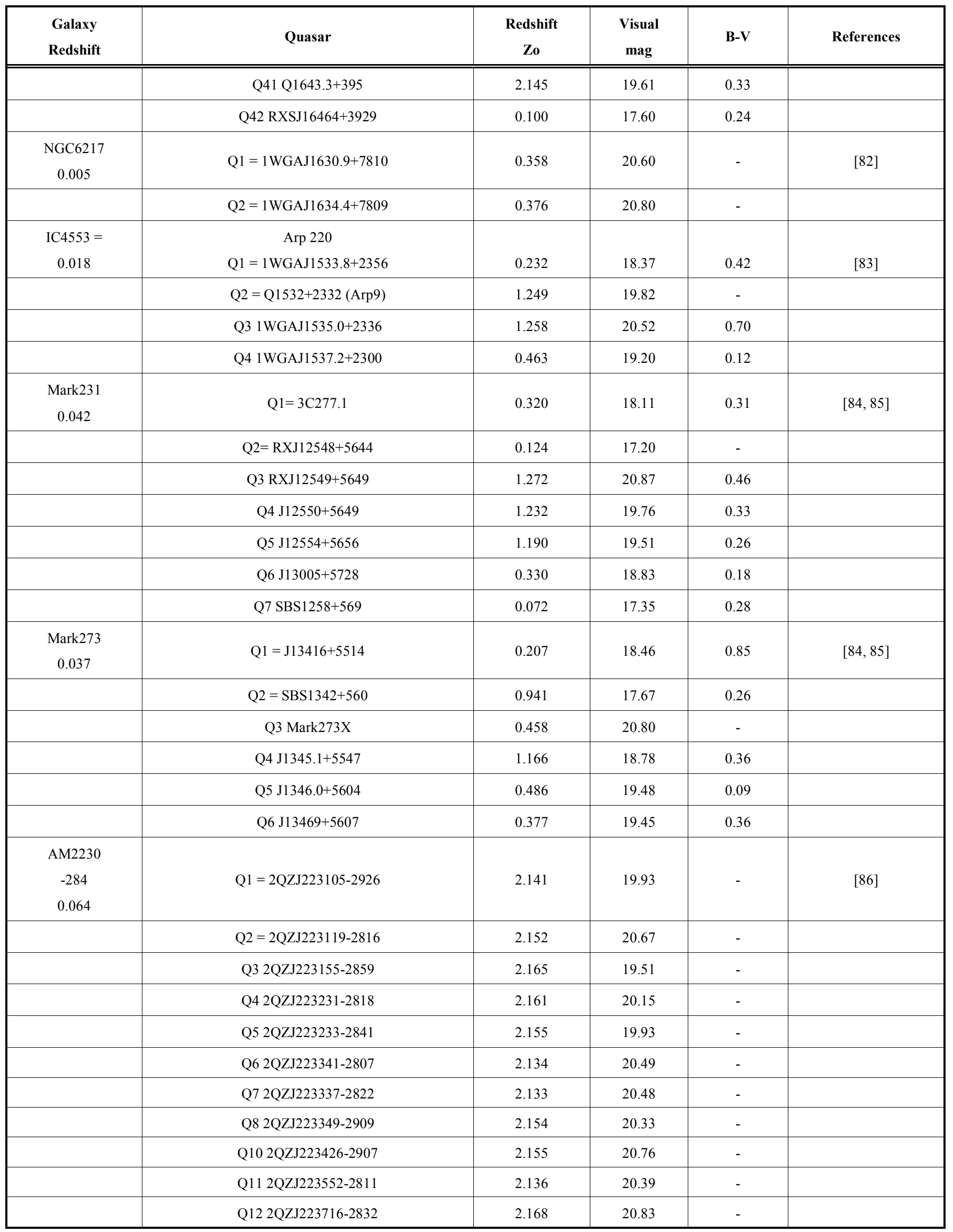


Table 1. contd....

\begin{tabular}{|c|c|c|c|c|c|}
\hline $\begin{array}{c}\text { Galaxy } \\
\text { Redshift }\end{array}$ & Quasar & $\begin{array}{c}\text { Redshift } \\
\text { Zo }\end{array}$ & $\begin{array}{c}\text { Visual } \\
\text { mag }\end{array}$ & B-V & References \\
\hline & Q13 2QZJ223755-2822 & 2.139 & 20.00 & - & \\
\hline & Q14 2QZJ223755-2901 & 2.137 & 20.36 & - & \\
\hline
\end{tabular}

tioned above, the cosmological redshift for a quasar near a galaxy is taken to be the redshift of this (parent) galaxy. So far, only groups of QSOs around low redshift galaxies have been studied, and the redshifts of the parent galaxies are taken as cosmological. With the eq (1), one could try to answer the above question, why modern redshift surveys could not confirm the Karlsson sequence? Modern surveys could probably contain predominantly more distant and faint QSOs. Their cosmological redshifts, according to the eq. (1), contribute substantially to the observed redshift. In other words, if a specific pattern (e.g. the Karlsson sequence) exists in the gravitational redshifts, it would be impossible to detect it, if the quasar sample includes a large number of distant quasars. The absence of positive finding in this case does not necessarily mean that the Karlsson sequence does not exist - it could be undetectable. The contribution by the $\left(1+z_{d}\right)$ term is also a "disturbing factor", however, Doppler shifts should be limited, not depending on distance. The positive finding of the Karlsson sequence in early surveys could be true, after all.

In $[18,59]$, the following procedure has been adopted in order to decompose redshifts of QSOs. First, for all quasars of a group around a low redshift galaxy, the redshift of the galaxy (assumed cosmological) is taken out from each quasar's observed redshift by:

$z_{i}=\left(z_{0}-z_{\text {gal }}\right) /\left(1+z_{\text {gal }}\right)$

This procedure assumes that all quasars of a specific group are at about the same distance as their parent galaxy. Each $z_{i}$ from eq (2) would now be composed by the gravitational and the Doppler component. As the projected Doppler component is limited (mostly less than $0.1 \mathrm{c}$, [59]), it seems possible to determine the gravitational reddening by simply comparing each $z_{i}$ value with the Karlsson sequence and take the nearest value from that sequence as $\mathrm{zg}_{\mathrm{gr}}$. Then the Doppler component could be decomposed by:

$\mathrm{z}_{\mathrm{d}}=\left(\mathrm{z}_{\mathrm{i}}-\mathrm{z}_{\mathrm{gr}}\right) /\left(1+\mathrm{z}_{\mathrm{gr}}\right)$

In the following, assumptions will be made in order to determine physical characteristics of local quasars. Here is the summary:

- the sample consists of groups of quasars, spatially associated with respective low redshift (parent) galaxy, according to published studies (see Table 1);

- the observed redshift of each quasar is considered to be composed by three components, according to eq.(1);

- the cosmological redshift component of each quasar is taken to be the redshift of the respective parent galaxy;
- quasars are single bodies and they have a thermal outer layer;

- the intrinsic redshift is due to gravitational reddening and for local quasars it is the largest component in each observed redshift;

- the gravitational redshifts are quantized, according to the Karlsson sequence.

The reality of these assumptions will be tested with the results and the relations obtained. Even one failure of the above assumptions will lead to inconsistent results. Radii of local quasars could be determined from:

$\log \left(\mathrm{r}_{\mathrm{q}} / \mathrm{r}_{\mathrm{o}}\right)=1 / 2 \cdot \log \left(\mathrm{L}_{\mathrm{q}} / \mathrm{L}_{\mathrm{o}}\right)+2 \log \left(\mathrm{T}_{\mathrm{o}} / \mathrm{T}_{\mathrm{q}}\right)$

In eq (4), r, L, and $T$ are the radius, luminosity, and the temperature, respectively. Symbols "q" and "o" stay for quasar and for the Sun, respectively. Implementation of this relation needs the assumption of a thermal outer layer. There is an additional question: do large redshifts introduce errors in the radii determination? Since a direct answer to this problem seems difficult, the same strategy will be applied again. Even one false assumption will lead to inconsistent results.

We could further determine the ratio $r_{g r} / r_{q}\left(r_{g r}\right.$ is the gravitational radius) from:

$\left(1+\mathrm{z}_{\mathrm{gr}}\right)=\left(1-\mathrm{r}_{\mathrm{gr}} / \mathrm{r}_{\mathrm{q}}\right)^{-1 / 2}$

Substituting respective $\mathrm{z}_{\mathrm{gr}}$ for each quasar and the $\mathrm{r}_{\mathrm{q}}$, we can get the quasar gravitational radius $\mathrm{r}_{\mathrm{gr}}$. The gravitational radius and the quasar mass $\mathrm{m}_{\mathrm{q}}$ are related by:

$\mathrm{r}_{\mathrm{gr}}=2 \mathrm{Gm}_{\mathrm{q}} / \mathrm{c}^{2}$

with $\mathrm{G}$ and $\mathrm{c}$ being the gravitational constant and the velocity of light. It is now possible to determine also the quasar density $\rho_{\mathrm{q}}$. Redshifts, magnitudes and colours for quasars are taken from Veron-Cetty and Veron, $13^{\text {th }}$ ed. [61]. In Table 2, the physical characteristics of the 116 sample quasars are listed (not included in [59]).

For all quasars with unknown $\mathrm{B}-\mathrm{V}$, quasar radii are determined from the absolute mag - radius relation [18]:

$\mathrm{M}_{\mathrm{q}}=48.099-4.318 . \log \mathrm{r}_{\mathrm{q}}$

In [18], the following density relation was established:

$\rho_{\mathrm{q}}=3 /(8 \pi) \cdot \mathrm{c}^{2} / \mathrm{G} \cdot 1 / \mathrm{r}_{\mathrm{q}}{ }^{2} \cdot\left\{1-1 /\left(1+\mathrm{z}_{\mathrm{gr}}\right)^{2}\right\}$

This is the relation between quasar density and its gravitational redshift. For local quasars, the gravitational redshift seems to be the main component of the observed redshift and it should be possible to compare computed density data for local quasars with eq (7). This comparison should provide also the test for validity of the assumptions made above. As eq (7) contains also the inverse square of quasar radius, it is 


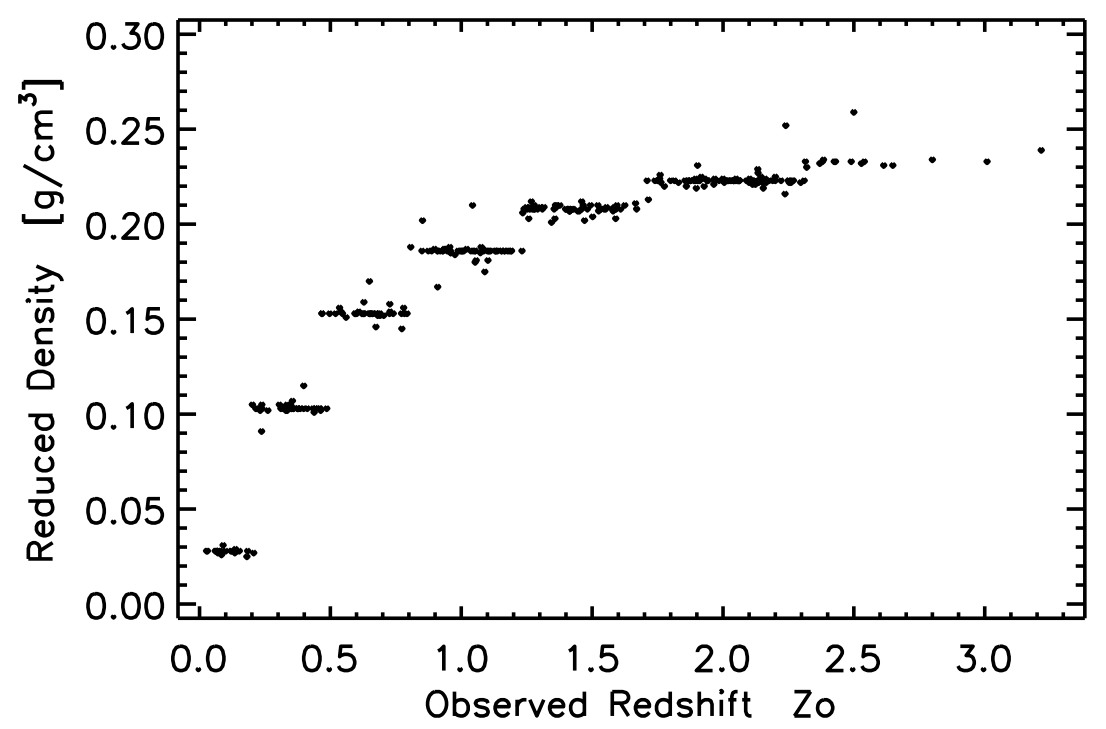

Fig. (1). "Reduced density- observed redshift" diagram for the sample of 341 quasars. All densities are reduced to a radius of $8.10^{13} \mathrm{~cm}$.

necessary to first reduce all density data to some radius of choice, e.g. $\mathrm{r}_{\mathrm{q}}=8.10^{13} \mathrm{~cm}$, in order to avoid the dependence on radius. This radius is arbitrary and already used in [18, 59]. The reduced density is:

$\rho^{\sim}=\left(\mathrm{r}_{\mathrm{q}} / 8 \cdot 10^{13}\right)^{2} \cdot \rho_{\mathrm{q}}$

With this substitution, eq (7) becomes:

$\rho \sim=3 /(8 \pi) \cdot c^{2} / G \cdot 1 /\left(8 \cdot 10^{13}\right)^{2} \cdot\left\{1-1 /\left(1+\mathrm{z}_{\mathrm{gr}}\right)^{2}\right\}$

This is the same as eq (7) but with a fixed radius $r_{q}=8$. $10^{13} \mathrm{~cm}$.

The choice of fixed radius is not essential for conclusions that follow. Data for the reduced densities are also listed in Table 2 and shown in Fig. (1) (altogether 341 QSOs). Densities are plotted versus the observed $\mathrm{z}_{\mathrm{o}}$ redshifts, although eq (9) should actually be plotted with the gravitational redshifts only. This causes a spread of the data in the $\mathrm{z}$ direction, which is tolerable for small cosmological redshifts (nearby parent galaxies). The reduced densities $\rho \sim$, corresponding to respective gravitational redshifts of the Karlsson sequence can be reckoned from eq (9):

Karlsson sequence of $\mathrm{z}_{\mathrm{gr}}: 0.06,0.30,0.60,0.96,1.41$, 1.96, 2.64, .. Reduced densities $\rho^{\sim}\left[\mathrm{g} / \mathrm{cm}^{3}\right] 0.028 ; 0.103$; $0.153 ; 0.186 ; 0.208 ; 0.223 ; 0.233$;

From Fig. (1), the observational data for all the 341 QSOs fit well to the eq (9). The agreement of observational data with the eq (9) could only be understood as confirmation of this procedure, including all the assumptions made above. The possibility of a coincidence seems very unlikely. More arguments against coincidence will be given below. Possible errors in Fig. (1), contributing to the scatter in the density, could be due to observational errors, variability of quasars, determination of radii from red-shifted spectra, or a projection of a distant (not local) quasar. We should also keep in mind that a part of the galaxy redshift could also have a non-cosmological origin, which could also contribute to errors. The scatter of data in the $\mathrm{z}$ - direction is simply due to the use of observed $z_{0}$ redshifts, instead of the gravitational $\mathrm{z}_{\mathrm{gr}}$ redshifts, as mentioned above.
If the relation on Fig (1) is real, as it seems, what could be the physics behind it? It is obvious that the SQM could not provide a clue. As pointed out in $[18,59]$ the relation on Fig. (1) could present evolution of quasars with decreasing density and a corresponding drop of the redshift (actually, only the gravitational component drops because of density). It looks, however, not to be a smooth, continuous transition, but a series of jumps to lower densities and corresponding redshift jumps to next lower value of the Karlsson sequence. This scenario has already been suggested by Arp [45]. Decreasing density could mean disintegration of matter. Such a possibility may seem exotic but I shall seriously take It into consideration and check possible consequences. Additional arguments of the "disintegration scenario" will be discussed below.

The density curve goes apparently to an asymptotic limit with increasing gravitational redshift [18]. As a consequence, at large redshifts only a small drop in density causes a large decrease in the gravitational redshift and respective drop in the observational redshift too. This could account for the decreasing number of QSOs with redshifts $z_{0}>3$ and for the absence of very large redshifts.

\section{THE LINEAR DENSITY EQUATION.}

From eq (5) it follows:

$\mathrm{r}_{\mathrm{gr}} / \mathrm{r}_{\mathrm{q}}=\left\{1-1 /\left(1+\mathrm{z}_{\mathrm{gr}}\right)^{2}\right\}$

Substituting $r_{\mathrm{gr}} / \mathrm{r}_{\mathrm{q}}$ in eq (7), we get:

$\rho_{\mathrm{q}}=3 /(8 \pi) \cdot \mathrm{c}^{2} / \mathrm{G} \cdot 1 / \mathrm{r}_{\mathrm{q}}{ }^{2} \cdot \mathrm{r}_{\mathrm{gr}} / \mathrm{r}_{\mathrm{q}}$

In eq (10), the quasar density also depends on the inverse square of radius. The same strategy could be applied also in this case by substituting $\rho^{\sim}$ from eq (8) to reduce the density to $\mathrm{r}_{\mathrm{q}}=8.10^{13} \mathrm{~cm}$. We get the simple linear density equation:

$\rho^{\sim}=3 /(8 \pi) \cdot c^{2} / G \cdot 1 /\left(8 \cdot 10^{13}\right)^{2} \cdot r_{g r} / r_{q}$

of the type: $\mathrm{y}=\mathrm{a}+\mathrm{bx}$, where $\mathbf{x}=\mathbf{r}_{\mathrm{gr}} / \mathbf{r}_{\mathbf{q}}, \mathrm{a}=0$ and $\mathrm{b}$ depends on the choice of radius in eq (8). For the following consideration this choice is not essential and I will keep the choice 
Table 2. Physical Characteristics of 116 Sample Quasars. Columns are: 1 - ID of Quasar, According to Table 1; 2 - Observed Redshift; 3 - Gravitational Redshift; 4 - Doppler shift; 5 - Absolute Magnitude; $6-\log \mathbf{r}_{\mathrm{q}}[\mathrm{cm}] ; 7-\log \mathrm{L}_{\mathrm{q}}[\mathrm{erg} / \mathrm{s}] ; 8-\log \mathbf{m}_{\mathrm{q}}[\mathrm{g}]$; 9 - Density $\rho_{q}\left[\mathrm{~g} / \mathrm{cm}^{3}\right] ; 10$ - Reduced Density $\left[\mathrm{g} / \mathrm{cm}^{3}\right]$ to Radius of $8.10^{13} \mathrm{~cm} ; 11$ - Ratio $\mathbf{r}_{\mathrm{gr}} / \mathbf{r}_{\mathrm{q}} ; 12$ - Quasar Mass in Units of $10^{6}$ Solar Masses

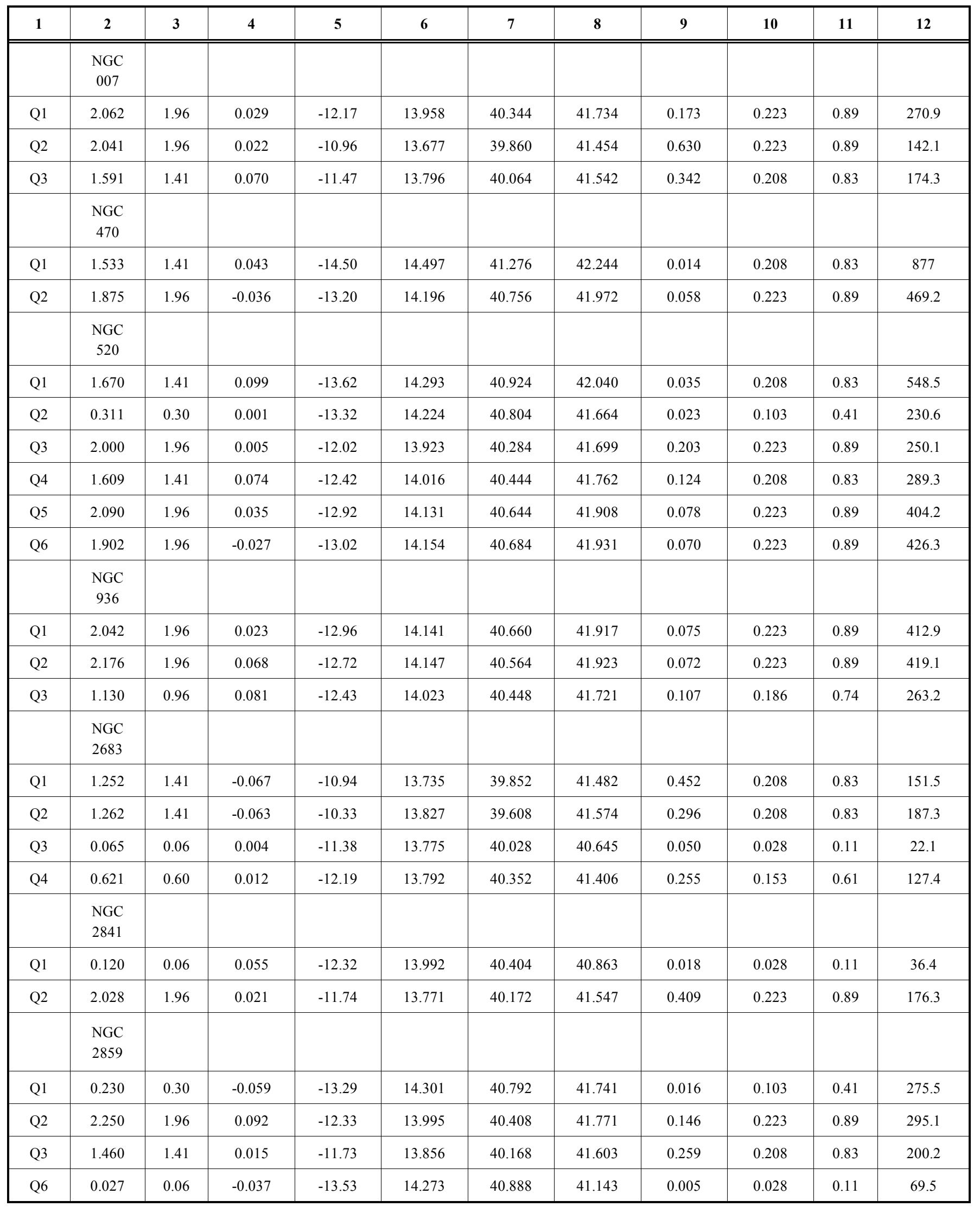


Table 2. contd...

\begin{tabular}{|c|c|c|c|c|c|c|c|c|c|c|c|}
\hline 1 & 2 & 3 & 4 & 5 & 6 & 7 & 8 & 9 & 10 & 11 & 12 \\
\hline & $\begin{array}{l}\text { NGC } \\
2916\end{array}$ & & & & & & & & & & \\
\hline Q1 & 1.546 & 1.41 & 0.044 & -13.81 & 14.346 & 41.000 & 42.093 & 0.027 & 0.208 & 0.83 & 619.0 \\
\hline Q2 & 0.238 & 0.30 & -0.059 & -13.84 & 14.344 & 41.012 & 41.784 & 0.013 & 0.103 & 0.41 & 304.3 \\
\hline Q4 & 1.868 & 1.96 & -0.043 & -13.69 & 14.066 & 40.952 & 41.842 & 0.105 & 0.223 & 0.89 & 347.6 \\
\hline \multirow[t]{2}{*}{ Q5 } & 1.279 & 1.41 & -0.066 & -13.95 & 14.451 & 41.056 & 42.198 & 0.017 & 0.208 & 0.83 & 788.8 \\
\hline & $\begin{array}{l}\text { NGC } \\
3184\end{array}$ & & & & & & & & & & \\
\hline Q3 & $\begin{array}{l}\text { NGC } \\
3384\end{array}$ & & & & & & & & & & \\
\hline Q1 & 0.442 & 0.30 & 0.077 & -10.94 & 13.588 & 39.852 & 41.028 & 0.439 & 0.103 & 0.41 & 53.3 \\
\hline Q2 & 1.280 & 1.41 & -0.081 & -10.98 & 13.770 & 39.868 & 41.517 & 0.384 & 0.208 & 0.83 & 164.6 \\
\hline Q4 & 1.107 & 0.96 & 0.044 & -11.19 & 13.710 & 39.952 & 41.408 & 0.453 & 0.186 & 0.74 & 127.9 \\
\hline Q5 & 1.192 & 0.96 & 0.086 & -10.25 & 13.513 & 39.576 & 41.211 & 1.122 & 0.186 & 0.74 & 81.3 \\
\hline Q8 & 1.134 & 0.96 & 0.057 & -11.69 & 14.015 & 40.152 & 41.713 & 0.111 & 0.186 & 0.74 & 258.1 \\
\hline Q1 & 2.100 & 1.96 & 0.038 & -14.45 & 14.486 & 41.256 & 42.262 & 0.015 & 0.222 & 0.89 & 914.0 \\
\hline Q2 & 1.399 & 1.41 & -0.013 & -12.95 & 14.138 & 40.656 & 41.885 & 0.071 & 0.208 & 0.83 & 383.8 \\
\hline Q3 & 0.930 & 0.96 & -0.024 & -12.95 & 14.138 & 40.656 & 41.836 & 0.063 & 0.186 & 0.74 & 342.9 \\
\hline Q4 & 0.690 & 0.60 & 0.047 & -13.85 & 14.347 & 41.016 & 41.960 & 0.020 & 0.153 & 0.61 & 456.5 \\
\hline Q5 & 0.328 & 0.30 & 0.012 & -12.75 & 14.092 & 40.576 & 41.532 & 0.043 & 0.103 & 0.41 & 170.1 \\
\hline \multirow[t]{2}{*}{ Q6 } & 1.710 & 1.96 & -0.093 & -14.35 & 14.462 & 41.216 & 42.239 & 0.017 & 0.223 & 0.89 & 866.5 \\
\hline & $\begin{array}{l}\text { NGC } \\
3842\end{array}$ & & & & & & & & & & \\
\hline Q1 & 0.335 & 0.30 & 0.005 & -16.21 & 14.893 & 41.960 & 42.333 & 0.001 & 0.102 & 0.41 & 1076.5 \\
\hline Q2 & 0.946 & 0.96 & -0.028 & -15.63 & 14.586 & 41.728 & 42.284 & 0.008 & 0.186 & 0.74 & 960.5 \\
\hline \multirow[t]{2}{*}{ Q3 } & 2.200 & 1.96 & 0.059 & -14.53 & 14.378 & 41.288 & 42.154 & 0.025 & 0.223 & 0.89 & 713.5 \\
\hline & $\begin{array}{l}\text { NGC } \\
6212\end{array}$ & & & & & & & & & & \\
\hline Q1 & 1.864 & 1.96 & -0.060 & -15.68 & 14.596 & 41.748 & 42.372 & 0.0092 & 0.223 & 0.89 & 1177.0 \\
\hline Q2 & 2.010 & 1.96 & -0.013 & -14.20 & 14.206 & 41.156 & 41.982 & 0.0553 & 0.223 & 0.89 & 479.6 \\
\hline
\end{tabular}


Table 2. contd...

\begin{tabular}{|c|c|c|c|c|c|c|c|c|c|c|c|}
\hline 1 & 2 & 3 & 4 & 5 & 6 & 7 & 8 & 9 & 10 & 11 & 12 \\
\hline Q3 & 1.898 & 1.96 & -0.049 & -15.87 & 14.835 & 41.824 & 42.611 & 0.003 & 0.219 & 0.89 & 2043.5 \\
\hline Q4 & 0.461 & 0.30 & 0.091 & -18.07 & 15.138 & 42.704 & 42.578 & 0.0004 & 0.103 & 0.41 & 1891.5 \\
\hline Q5 & 0.143 & 0.06 & 0.047 & -17.12 & 15.085 & 42.324 & 41.955 & 0.0001 & 0.028 & 0.11 & 451.3 \\
\hline Q6 & 0.030 & 0.06 & -0.057 & -17.70 & 15.238 & 42.556 & 42.109 & 0.0001 & 0.028 & 0.11 & 642.0 \\
\hline Q7 & 1.965 & 1.96 & -0.027 & -16.30 & 14.914 & 41.996 & 42.690 & 0.0021 & 0.221 & 0.89 & 2451.0 \\
\hline Q8 & 1.183 & 0.96 & 0.081 & -15.10 & 14.443 & 41.516 & 42.141 & 0.016 & 0.186 & 0.74 & 692.0 \\
\hline Q9 & 1.666 & 1.41 & 0.074 & -16.13 & 14.875 & 41.928 & 42.622 & 0.0024 & 0.211 & 0.83 & 2092.0 \\
\hline Q10 & 2.253 & 1.96 & 0.067 & -16.42 & 14.684 & 42.044 & 42.460 & 0.0061 & 0.222 & 0.89 & 1441.5 \\
\hline Q11 & 1.625 & 1.41 & 0.058 & -16.90 & 14.780 & 42.236 & 42.527 & 0.0037 & 0.210 & 0.83 & 1680.7 \\
\hline Q12 & 2.614 & 2.64 & -0.036 & -16.41 & 14.648 & 42.040 & 42.442 & 0.0075 & 0.231 & 0.92 & 1384.9 \\
\hline Q13 & 0.540 & 0.60 & -0.066 & -15.67 & 14.522 & 41.744 & 42.136 & 0.009 & 0.154 & 0.61 & 683.5 \\
\hline Q14 & 1.005 & 0.96 & -0.007 & -17.45 & 14.950 & 42.456 & 42.648 & 0.002 & 0.186 & 0.74 & 2221.0 \\
\hline Q15 & 0.625 & 0.60 & -0.014 & -14.99 & 14.252 & 41.472 & 41.865 & 0.031 & 0.153 & 0.61 & 366.8 \\
\hline Q16 & 2.529 & 2.64 & -0.059 & -15.18 & 14.610 & 41.548 & 42.405 & 0.009 & 0.232 & 0.92 & 1271.0 \\
\hline Q17 & 1.860 & 1.96 & -0.062 & -16.65 & 14.923 & 42.136 & 42.700 & 0.002 & 0.220 & 0.89 & 2504.5 \\
\hline Q18 & 1.580 & 1.41 & 0.039 & -14.65 & 14.495 & 41.336 & 42.242 & 0.0136 & 0.207 & 0.83 & 872.5 \\
\hline Q19 & 1.595 & 1.41 & 0.045 & -15.88 & 14.661 & 41.828 & 42.408 & 0.0064 & 0.210 & 0.83 & 1278.0 \\
\hline Q20 & 1.466 & 1.41 & -0.007 & -16.03 & 14.780 & 41.888 & 42.527 & 0.0037 & 0.210 & 0.83 & 1683.5 \\
\hline Q21 & 1.414 & 1.41 & -0.027 & -15.50 & 14.665 & 41.676 & 42.412 & 0.0062 & 0.207 & 0.83 & 1291.0 \\
\hline Q22 & 2.384 & 2.64 & -0.098 & -16.23 & 14.681 & 41.968 & 42.476 & 0.0065 & 0.234 & 0.92 & 1496.0 \\
\hline Q23 & 0.595 & 0.60 & -0.032 & -18.91 & 15.217 & 43.040 & 42.831 & 0.0004 & 0.153 & 0.61 & 3387.5 \\
\hline Q24 & 1.360 & 1.41 & -0.049 & -17.26 & 14.937 & 42.380 & 42.684 & 0.0018 & 0.210 & 0.83 & 2413.0 \\
\hline Q25 & 2.000 & 1.96 & -0.016 & -15.49 & 14.498 & 41.672 & 42.274 & 0.0144 & 0.223 & 0.89 & 939.5 \\
\hline Q26 & 2.260 & 1.96 & 0.069 & -15.48 & 14.680 & 41.668 & 42.456 & 0.0062 & 0.222 & 0.89 & 1428.5 \\
\hline Q27 & 2.000 & 1.96 & -0.016 & -14.68 & 14.434 & 41.348 & 42.210 & 0.0194 & 0.223 & 0.89 & 811.0 \\
\hline Q28 & 0.443 & 0.30 & 0.078 & -16.20 & 14.362 & 41.956 & 41.802 & 0.0124 & 0.103 & 0.41 & 317.2 \\
\hline Q29 & 0.704 & 0.60 & 0.034 & -17.18 & 14.749 & 42.348 & 42.362 & 0.0031 & 0.152 & 0.61 & 1151.5 \\
\hline Q30 & 1.083 & 0.96 & 0.032 & -16.44 & 14.773 & 42.052 & 42.471 & 0.0034 & 0.187 & 0.74 & 1478.0 \\
\hline Q31 & 2.113 & 1.96 & 0.021 & -16.19 & 14.698 & 41.952 & 42.474 & 0.0057 & 0.221 & 0.89 & 1488.5 \\
\hline Q32 & 0.594 & 0.60 & -0.033 & -16.00 & 14.426 & 41.876 & 42.040 & 0.014 & 0.153 & 0.61 & 547.5 \\
\hline Q33 & 1.358 & 1.41 & -0.050 & -16.95 & 14.983 & 42.256 & 42.730 & 0.0014 & 0.203 & 0.83 & 2687.0 \\
\hline Q34 & 0.434 & 0.30 & 0.071 & -16.11 & 14.599 & 41.920 & 42.039 & 0.004 & 0.103 & 0.41 & 546.5 \\
\hline Q35 & 1.377 & 1.41 & -0.042 & -16.05 & 14.747 & 41.896 & 42.494 & 0.0043 & 0.210 & 0.83 & 1560.5 \\
\hline Q36 & 0.608 & 0.60 & -0.024 & -16.37 & 14.438 & 42.024 & 42.052 & 0.013 & 0.154 & 0.61 & 563.0 \\
\hline Q37 & 1.268 & 1.41 & -0.086 & -16.19 & 14.964 & 41.952 & 42.711 & 0.0016 & 0.212 & 0.83 & 2570.0 \\
\hline Q39 & 1.451 & 1.41 & -0.012 & -16.46 & 14.669 & 42.060 & 42.416 & 0.006 & 0.207 & 0.83 & 1302.0 \\
\hline Q40 & 1.877 & 1.96 & -0.056 & -16.01 & 14.500 & 41.880 & 42.276 & 0.0143 & 0.223 & 0.89 & 943.5 \\
\hline
\end{tabular}


Table 2. contd...

\begin{tabular}{|c|c|c|c|c|c|c|c|c|c|c|c|}
\hline 1 & 2 & 3 & 4 & 5 & 6 & 7 & 8 & 9 & 10 & 11 & 12 \\
\hline Q41 & 2.145 & 1.96 & 0.031 & -15.89 & 14.743 & 41.832 & 42.519 & 0.0047 & 0.225 & 0.89 & 1653.0 \\
\hline \multirow[t]{2}{*}{ Q42 } & 0.100 & 0.06 & 0.008 & -17.90 & 15.040 & 42.636 & 41.910 & 0.0002 & 0.028 & 0.11 & 406.4 \\
\hline & $\begin{array}{l}\text { Mark } \\
231\end{array}$ & & & & & & & & & & \\
\hline Q1 & 0.320 & 0.30 & -0.025 & -18.15 & 15.176 & 42.736 & 42.616 & 0.0003 & 0.103 & 0.41 & 2067.0 \\
\hline Q2 & 0.124 & 0.06 & 0.018 & -19.06 & 15.553 & 43.100 & 42.424 & $1 \times 10^{-5}$ & 0.028 & 0.11 & 1326.0 \\
\hline Q3 & 1.272 & 1.41 & -0.095 & -15.39 & 14.763 & 41.632 & 42.510 & 0.0040 & 0.208 & 0.83 & 1617.5 \\
\hline Q4 & 1.232 & 0.96 & 0.093 & -16.50 & 14.865 & 42.076 & 42.563 & 0.0022 & 0.186 & 0.74 & 1827.5 \\
\hline Q5 & 1.190 & 0.96 & 0.072 & -16.75 & 14.835 & 42.176 & 42.533 & 0.0026 & 0.186 & 0.74 & 1705.0 \\
\hline Q6 & 0.330 & 0.30 & -0.018 & -17.43 & 14.874 & 42.448 & 42.314 & 0.0012 & 0.102 & 0.41 & 1030.5 \\
\hline \multirow[t]{2}{*}{ Q7 } & 0.072 & 0.06 & -0.029 & -18.91 & 15.293 & 43.040 & 42.163 & $5 \times 10^{-5}$ & 0.028 & 0.11 & 727.5 \\
\hline & $\begin{array}{l}\text { Mark } \\
273\end{array}$ & & & & & & & & & & \\
\hline Q1 & 0.207 & 0.06 & 0.098 & -17.65 & 15.505 & 42.536 & 42.375 & $2 \times 10^{-5}$ & 0.027 & 0.11 & 1187.0 \\
\hline Q2 & 0.941 & 0.96 & -0.045 & -18.44 & 15.173 & 42.852 & 42.871 & 0.0005 & 0.187 & 0.74 & 3712.5 \\
\hline Q3 & 0.458 & 0.30 & 0.082 & -15.31 & 14.685 & 41.600 & 42.125 & 0.0028 & 0.103 & 0.41 & 666.0 \\
\hline Q4 & 1.166 & 0.96 & 0.066 & -17.33 & 15.059 & 42.408 & 42.757 & 0.0009 & 0.186 & 0.74 & 2860.0 \\
\hline Q5 & 0.486 & 0.30 & 0.102 & -16.63 & 14.594 & 42.128 & 42.034 & 0.0043 & 0.103 & 0.41 & 540.5 \\
\hline \multirow[t]{2}{*}{ Q6 } & 0.377 & 0.30 & 0.022 & -16.66 & 14.926 & 42.140 & 42.365 & 0.0009 & 0.103 & 0.41 & 1159.5 \\
\hline & $\begin{array}{c}\text { AM } \\
2230-284\end{array}$ & & & & & & & & & & \\
\hline Q1 & 2.141 & 1.96 & -0.003 & -17.14 & 15.109 & 42.332 & 42.885 & 0.0009 & 0.223 & 0.89 & 3835.5 \\
\hline Q2 & 2.152 & 1.96 & 0.001 & -16.40 & 14.937 & 42.036 & 42.713 & 0.0019 & 0.222 & 0.89 & 2585.0 \\
\hline Q3 & 2.165 & 1.96 & 0.005 & -17.56 & 15.206 & 42.500 & 42.982 & 0.0006 & 0.222 & 0.89 & 4798.5 \\
\hline Q4 & 2.161 & 1.96 & 0.004 & -16.92 & 15.058 & 42.244 & 42.834 & 0.0011 & 0.224 & 0.89 & 3411.0 \\
\hline Q5 & 2.155 & 1.96 & 0.002 & -17.14 & 15.109 & 42.332 & 42.885 & 0.0009 & 0.222 & 0.89 & 3835.5 \\
\hline Q6 & 2.134 & 1.96 & -0.005 & -16.58 & 14.979 & 42.108 & 42.755 & 0.0016 & 0.227 & 0.89 & 2845.5 \\
\hline Q7 & 2.133 & 1.96 & -0.005 & -16.59 & 14.981 & 42.112 & 42.758 & 0.0016 & 0.229 & 0.89 & 2860.5 \\
\hline Q8 & 2.154 & 1.96 & 0.001 & -16.74 & 15.016 & 42.172 & 42.792 & 0.0013 & 0.219 & 0.89 & 3099.0 \\
\hline Q10 & 2.155 & 1.96 & 0.002 & -16.31 & 14.916 & 42.000 & 42.693 & 0.0021 & 0.223 & 0.89 & 2464.0 \\
\hline Q11 & 2.136 & 1.96 & -0.004 & -16.68 & 15.002 & 42.148 & 42.778 & 0.0014 & 0.222 & 0.89 & 3001.5 \\
\hline Q12 & 2.168 & 1.96 & 0.006 & -16.24 & 14.900 & 41.972 & 42.676 & 0.0023 & 0.223 & 0.89 & 2373.5 \\
\hline Q13 & 2.139 & 1.96 & -0.003 & -17.07 & 15.092 & 42.304 & 42.869 & 0.0009 & 0.223 & 0.89 & 3695.0 \\
\hline Q14 & 2.137 & 1.96 & -0.004 & -16.71 & 15.009 & 42.160 & 42.785 & 0.0014 & 0.223 & 0.89 & 3049.8 \\
\hline
\end{tabular}

in eq (8) and in eq (11). Then in the [cm, g, s] system, b = 0.251549 in eq (11). We therefore get:

$\rho \sim=0.251549 . \mathrm{rgr}_{\mathrm{gr}} / \mathrm{r}_{\mathrm{q}}$
The sample of 341 QSOs (Table 2, this study, and [59]) are plotted in Fig. (2). The straight line is obvious, and the coefficients are: $a=0.0002$ and $b=0.251$. Let me now turn back to the question: could this be a coincidence? Although 


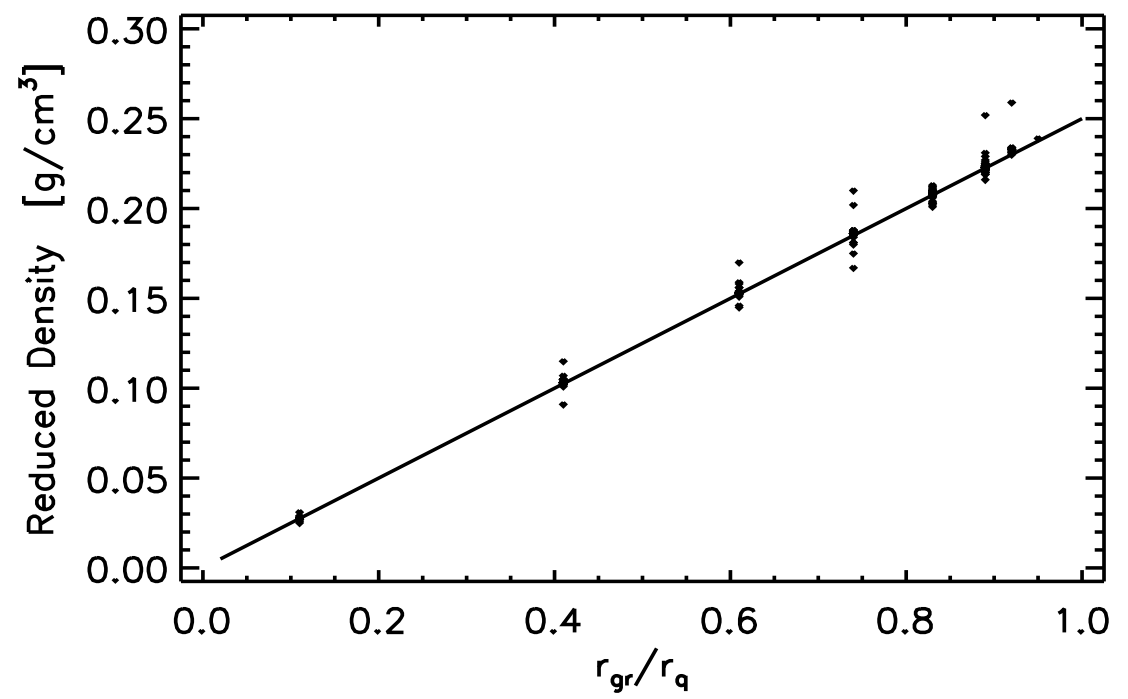

Fig. (2). The linear relation of quasar reduced density with $r_{g r} / r_{q}$ for the sample of 341 QSOs. The mean line equation is: $\rho^{\sim}=0.0002+0.251$ . $\mathrm{r}_{\mathrm{gr}} / \mathrm{r}_{\mathrm{q}}$ (see text).

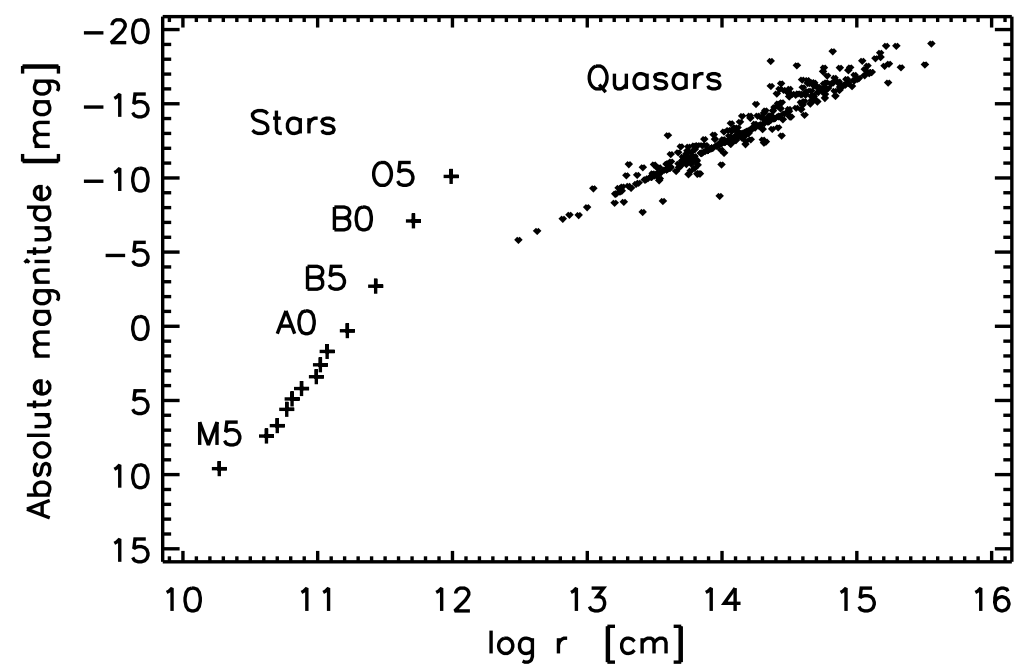

Fig. (3). The relation "absolute magnitude - radius" for 341 sample quasars (dots). The same relation is shown also for stars (crosses), as mean values for $\mathrm{O} 5, \mathrm{~B} 0, . .,, \mathrm{M} 5$.

very unlikely, both relations, on Fig. (1) and on Fig. (2) considered separately could be due to a coincidence. However, there is now an additional argument. How is it possible that these two "coincidences" are related? Their relation is obvious. The eq (12) follows from eq (7). Coincidences can not be related, or else they are not coincidences. In the following, additional arguments will be presented for the credibility of the procedure. Reducing densities to a different radius will produce a different slope coefficient in eq (12) but this is not essential for the results and the conclusions. The slight deviation of coefficients " $a$ " and " $b$ " from the respective theoretical values in eq (12) are likely to be due to observational uncertainties. As will be shown, the linear density equation (12) could be applied also to other structures.

\section{SOME RELATIONS FOR QUASARS}

In Fig. (3), the absolute magnitude is plotted versus radius for the sample of 341 QSOs. The sequence of stars is also shown as mean values for $\mathrm{O} 5, \mathrm{~B} 0, \mathrm{~B} 5, \mathrm{~A} 0, \ldots . ., \mathrm{M} 5$.
There is a good agreement with the respective relation in [18, 59]. Therefore, by increasing the QSOs sample this relation is confirmed.

In Fig. (4), the absolute magnitude is plotted against the mass for 341 sample QSOs. Shown is also the sequence of stars. There is a good agreement with the respective relation from $[18,59]$.

The "mass-radius" relation is shown in Fig. (5) for the sample of 341 QSOs. The sequence of stars is also shown. The agreement with $[18,59]$ is good. It has already been pointed out in $[18,59]$ that this "mass-radius" relation implies that fainter quasars have larger gravitational redshifts (leading to larger observed redshifts), which has been discussed already by Greenstein and Schmidt [6]. The mean "mass-radius" relation for 341 sample QSOs is:

$\log \mathrm{m}_{\mathrm{q}}=28.67+0.93 \log \mathrm{r}_{\mathrm{q}}$ 


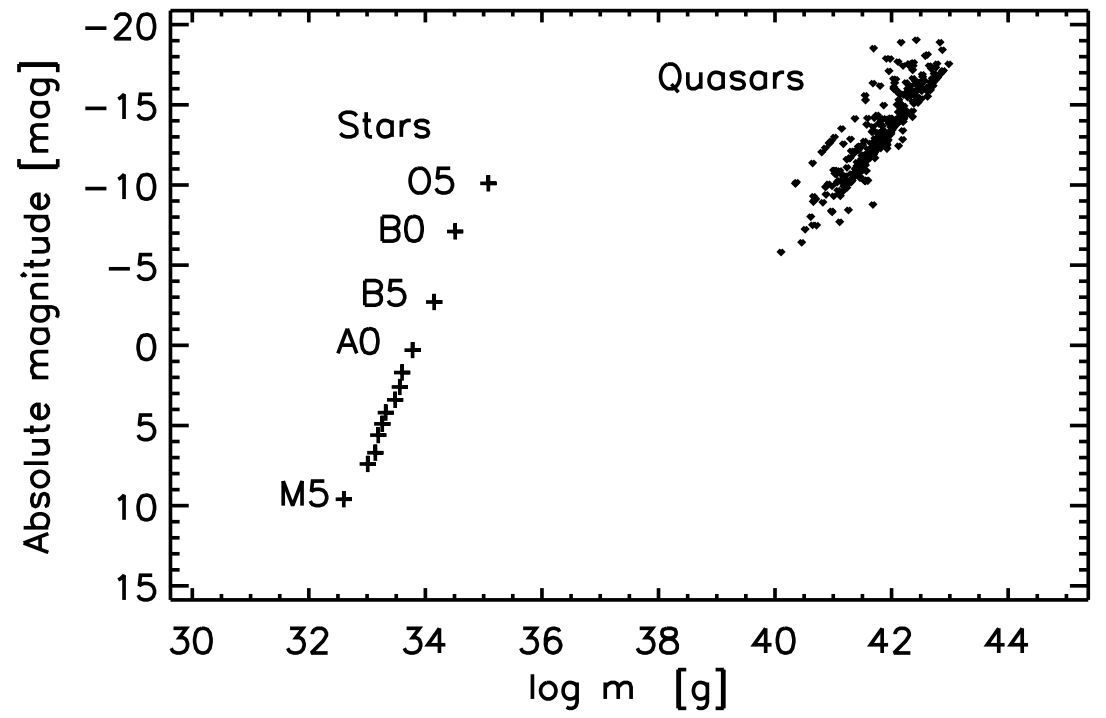

Fig. (4). The relation "absolute magnitude - mass" for 341 sample quasars (dots). The same relation is shown also for stars (crosses), as mean values for $\mathrm{O} 5, \mathrm{~B} 0, . ., \mathrm{M} 5$.

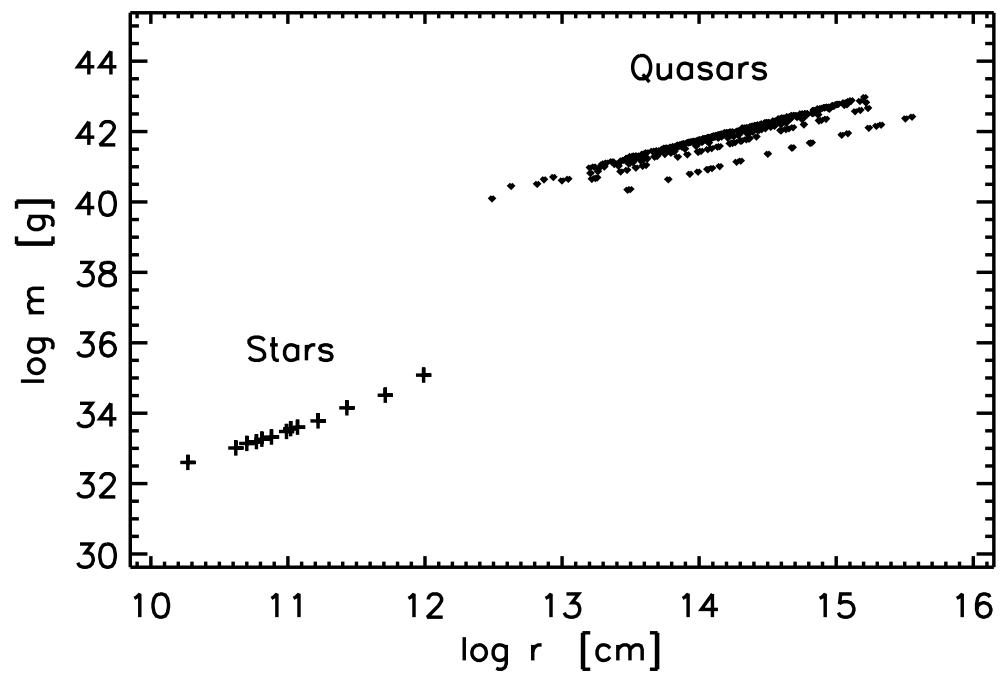

Fig. (5). The relation "mass - radius" for 341 sample quasars (dots). The same relation is shown also for stars (crosses), as mean values for $\mathrm{O} 5, \mathrm{~B} 0, \mathrm{~B} 5, \ldots, \mathrm{M} 5$.

The "mass-luminosity" relation is shown in Fig. (6) for the sample of 341 QSOs.

The stellar sequence is also shown. The mean "massluminosity" relation for 341 QSOs is:

$\log \mathrm{L}_{\mathrm{q}}=-27.49+1.63 . \log \mathrm{m}_{\mathrm{q}}$

in agreement with $[18,59]$. The physics behind these relations is not yet clear.

\section{EVIDENCE OF EVOLUTION OF QUASARS.}

Possible evolutionary scenario has already been mentioned by the discussion of Fig. (1). Quasars seem to evolve with decreasing redshifts because of decreasing density. From that scenario, other relations could also be expected and they will be looked for in this section. In the first place, evolutionary effects could be searched for by comparing groups of quasars at different distances. This is shown in Fig. (7) and Fig. (8), where plots of quasar absolute magnitude
(Fig (7)) and quasar mass (Fig. 8) versus respective redshift of parent galaxy are presented. Both the quasar absolute magnitude and the quasar mass increase with the cosmological redshift, i.e. with distance $\left(z_{\mathrm{gal}}=\mathrm{z}_{\mathrm{c}}\right)$.

As pointed out in $[18,59]$, in order to study evolutionary effects on luminosity with decreasing gravitational redshift, it was necessary to sample QSOs in different groups (see Fig. (3) in [59]). It is now apparent that this sampling corresponds to the respective redshift of parent galaxy, i.e. to the cosmological quasar redshift. As apparent from Fig. (7) and Fig. (8), at earlier stages of the Universe, at least to a distance, corresponding to $\mathrm{z}_{\mathrm{gal}}=0.064$, the quasars seem to have larger masses and larger luminosities. The reason for that remains unclear. Especially strong is the dependence of luminosity and mass of QSOs in the parts of the diagrams below cosmological redshift of about 0.003 . Apparently, if a study of evolutionary effects of mass and luminosity of quasars due to $\mathbf{z}_{\mathrm{gr}}$ is to be correct, the dependence on distance 


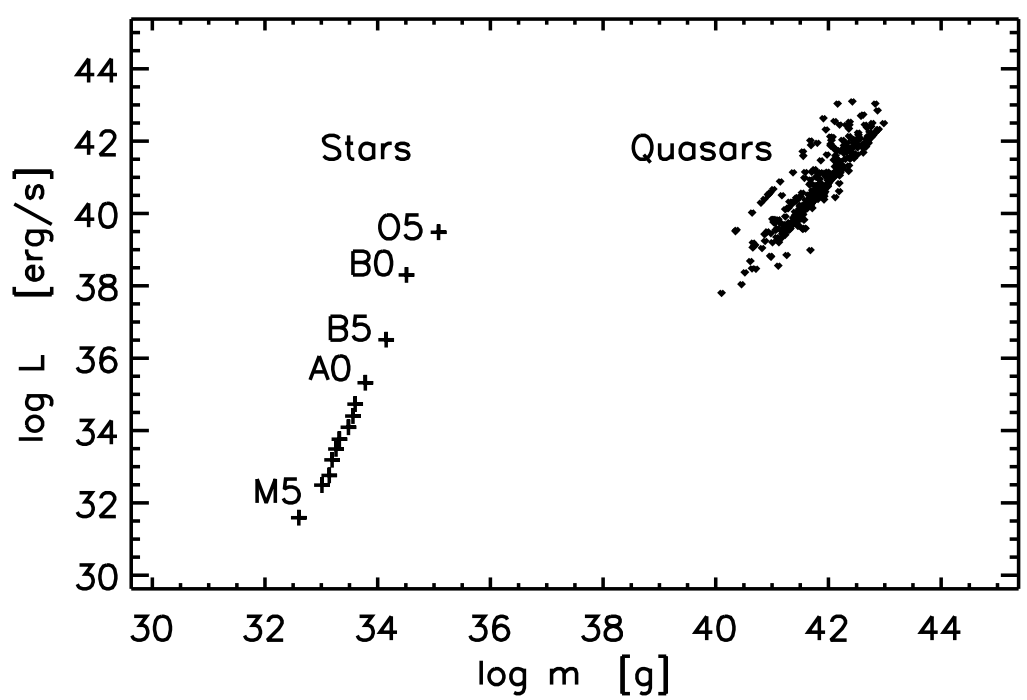

Fig. (6). The "mass-luminosity" relation for 341 sample quasars (dots). The same relation is shown also for stars (crosses), as mean values for O5, B0, B5,.., M5.

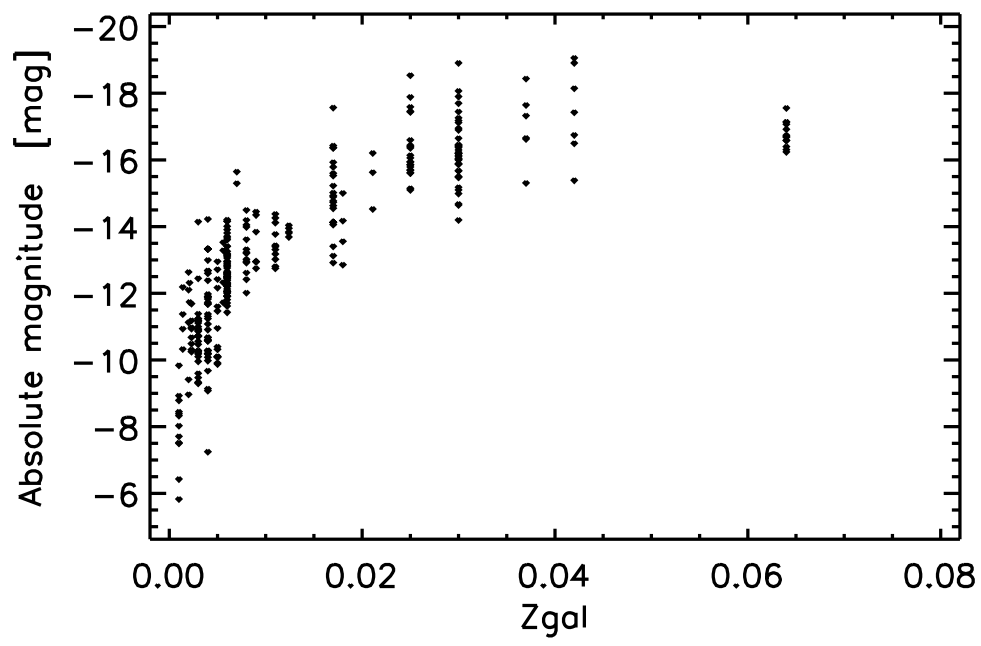

Fig. (7). Dependence of quasar absolute magnitude on distance for 341 sample QSOs. Note the increasing "mean" luminosity to about $\mathrm{z}_{\mathrm{gal}}=$ 0.025 .

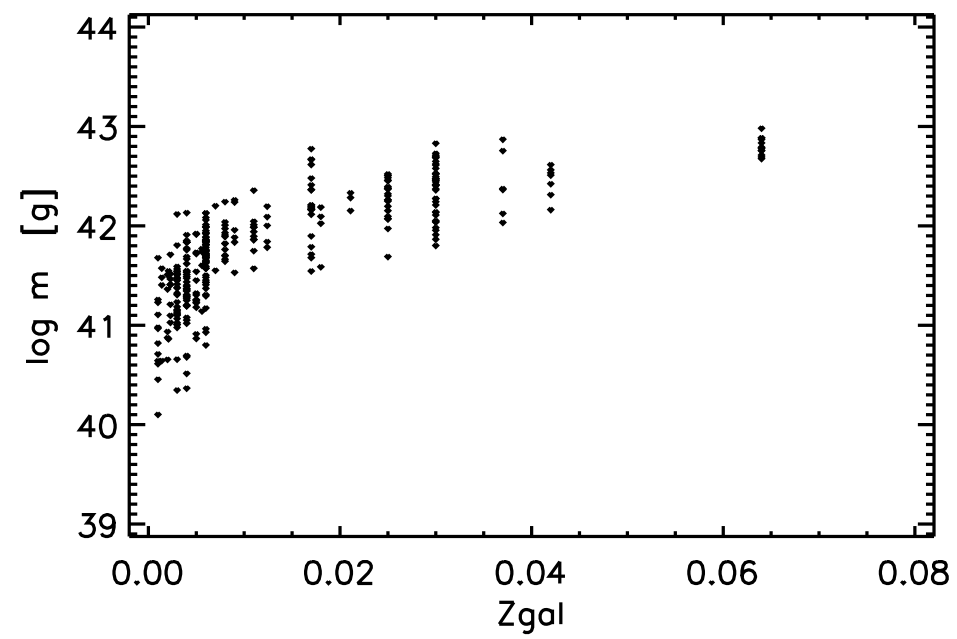

Fig. (8). Dependence of quasar mass on distance for 341 sample QSOs. Note the increasing "mean" mass to about $z_{\text {gal }}=0.025$. 


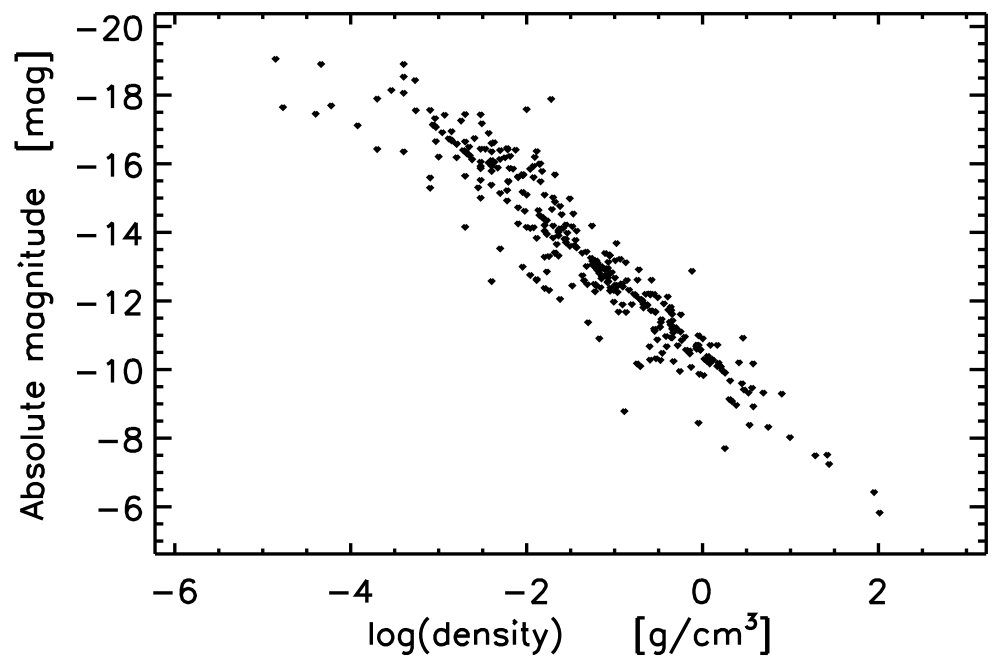

Fig. (9). Relation "absolute magnitude - density" for 341 sample quasars.

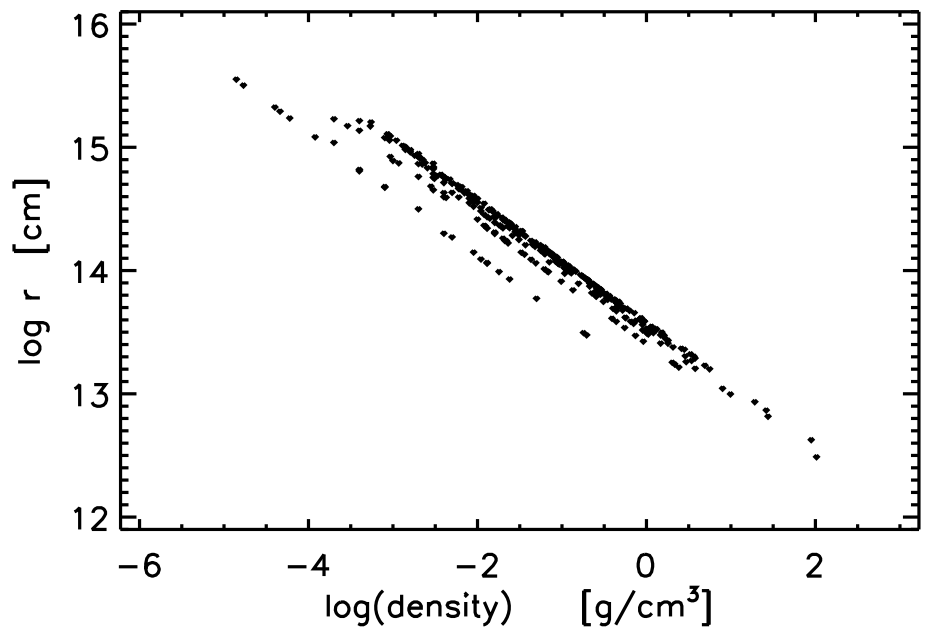

Fig. (10). Relation "radius - density" for 341 sample quasars.

should be taken into account. Evolution with decreasing density and preserved mass of quasar would imply possible relation between quasar density and radius. In [18, 59], an evolutionary increase of quasar luminosity and radius was suggested. Looking for further evidence of evolutionary effects, in Fig. (9) is plotted the quasar absolute magnitude versus quasar density. From Fig. (9), the apparent relation is in the sense that larger luminosities correspond to lower density (the presumed direction of evolution).

From Fig. (9), in direction of increasing density, quasars get less luminous. This relation prompts further tantalizing questions. Is it possible to extend the relation to even larger densities? If yes, could this relation have a bearing on the much discussed problem of the hidden (dark) mass? Is it possible that the "dark" masses could be faint because of high density?

In Fig. (10), quasar radius is plotted versus quasar density. The obvious trend of increasing radius by decreasing density is consistent with the disintegration hypothesis.

Quasar absolute magnitude (Fig. 11) and quasar radius (Fig. 12) are plotted versus quasar redshift $z_{0}$, as a check for consistency. The presumed direction of evolution is decreasing redshift. On these diagrams, as well as on Fig. (13), quasars with cosmological redshift less than 0.003 are not included, in order to partly avoid respective dependence on distance. The large scatter on these plots is possibly due to the still remaining effects of distance dependence. Yet, the absolute magnitude and the radius seem to be possibly increasing with decreasing redshift, according to the evolutionary scenario.

An interesting question is, are there any evolutionary effects observed in the quasar masses? According to the Arp's scenario [45], quasars develop into galaxies. Building a stellar population around a quasar requires (if the total mass is preserved) that at some stage of the evolution quasars have to start losing mass. Is there any evidence of this process? In Fig. (13) quasar mass is plotted versus the redshift. The interpretation of this diagram is not straight. There could be a mass drop at the late stages of evolution, for $z_{0}<0.60$ but this matter needs further study.

In Fig. (14), a plot of the quasar density versus mass is presented. The apparent trend confirms respective results from $[18,59]$. Larger masses correspond to smaller densities. 


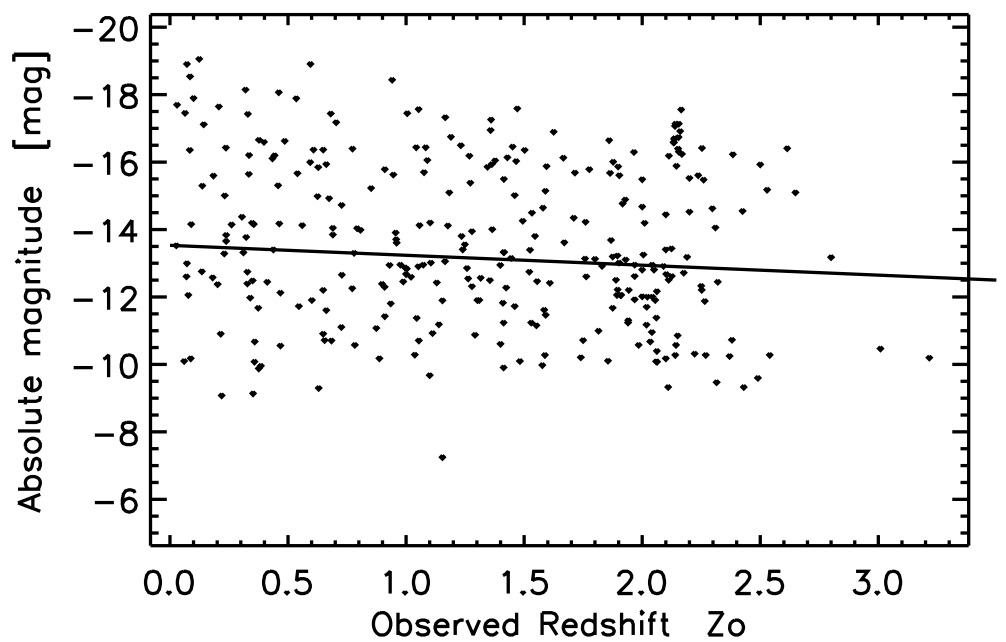

Fig. (11). Plot of quasar absolute magnitudes versus observed redshift for the sample quasars with $z_{\text {gal }}>0.003\left(z_{\text {gal }}=z_{c}\right.$, see text).

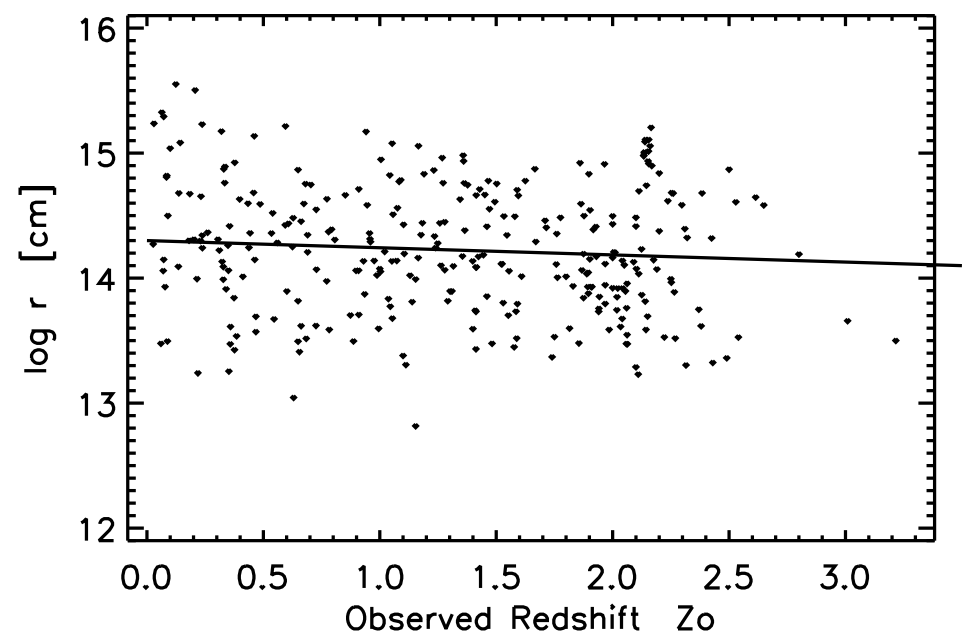

Fig. (12). Plot of quasar radius versus observed redshift for the sample quasars with $z_{\mathrm{gal}}>0.003\left(\mathrm{z}_{\mathrm{gal}}=\mathrm{z}_{\mathrm{c}}\right.$, see text).

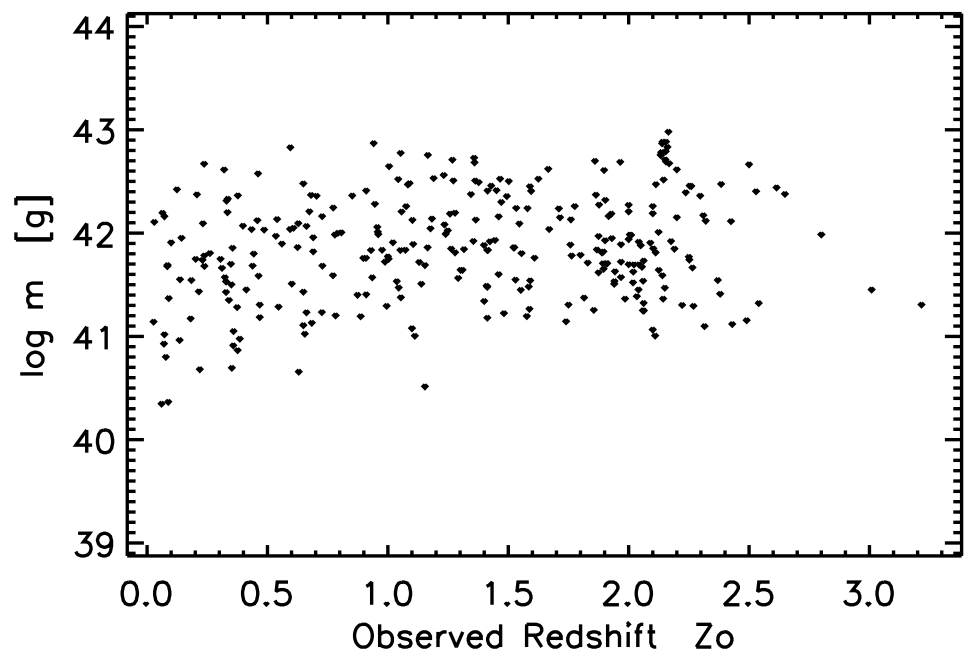

Fig. (13). Plot of quasar mass versus observed redshift for quasars with $\mathrm{z}_{\text {gal }}>0.003$.

If the presumed direction of evolution is towards decreasing densities, the relation on Fig. (14) shows that quasars with larger masses evolve more rapidly.

The concept of evolution due to disintegration of some mysterious dense matter is not a new one. It has been intro- duced first by Victor Ambartsumian about 60 y ago [87]. It now appears that this concept gets new impetus. The evidence presented in this section seems to support possible consequences of the evolutionary scenario with decreasing density. 


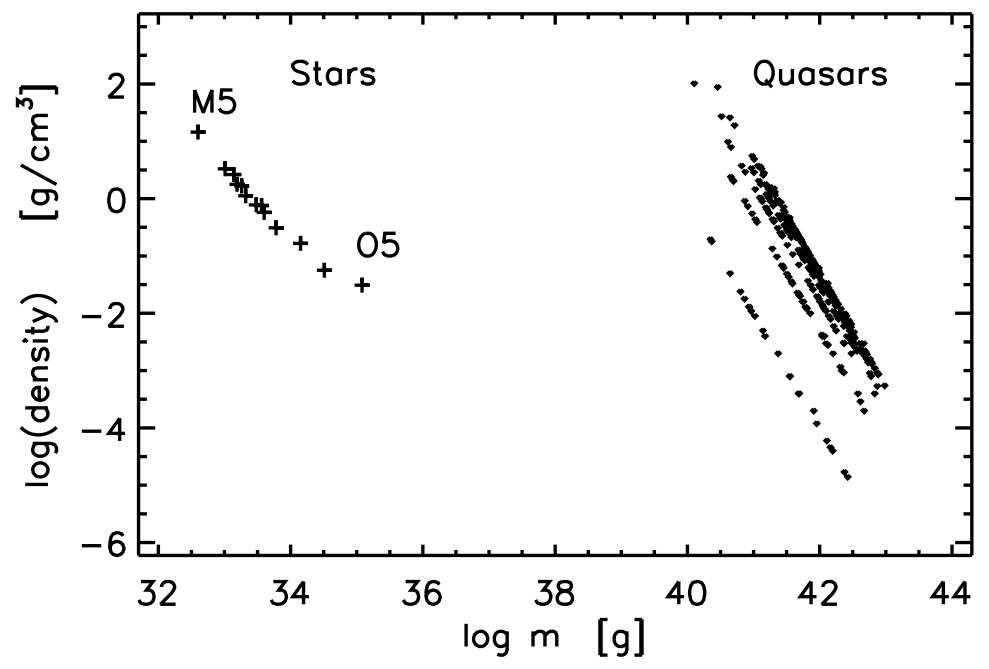

Fig. (14). Relation "mass - density" for the sample of 341 quasars (dots). The same relation is shown for stars (crosses), as mean values for $\mathrm{O} 5, \mathrm{~B} 0, \mathrm{~B} 5, \ldots ., \mathrm{M} 5$.

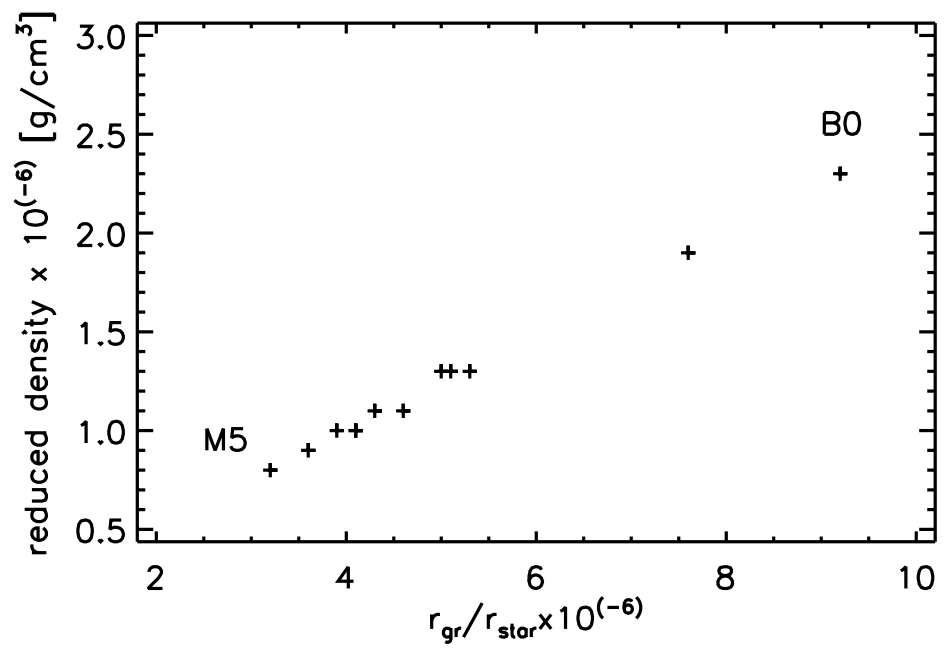

Fig. (15). The linear relation of stellar reduced density (to a radius of $8.10^{13} \mathrm{~cm}$ ). with the stellar rgr/rstar. The sequence is: B0, B5, A0, F0, A5, F5, G5, G0, K0, M0, M5. The linear equation is: $\rho \sim=-2.10^{-9}+0.2505 \cdot \mathrm{r}_{\mathrm{gr}} / \mathrm{r}_{\text {star }}$.

\section{IS THERE A QUASAR-STELLAR CONNECTION?}

In the previous sections, on Figs. (3-6), and on Fig. (14) the sequence of stars along with quasars were shown. There is a striking similarity when stars and quasars are compared on these figures, although respective relations for stars and quasars are not identical. But what if there would be a relation, identical for quasars and stars? On Fig. (15), the reduced stellar densities (to the same radius of $8.10^{13} \mathrm{~cm}$ ) are plotted versus the ratio $r_{g r} / r_{\text {star }}$. This plot corresponds to the plot on Fig. (2) for quasars. Surprisingly, the straight line in Fig. (15) corresponds closely to the linear density equation (12), and the coefficients are: $a=-2.10^{-9}, b=0.2505$ (correlation coeff is 0.998). Comparing with respective values for quasars, this is probably the same relation. Deviation of the coeff "a" from zero, and deviation of coeff " $b$ " from the theoretical value 0.251549 are probably due to observational uncertainties in the case of stars, as well as for quasars. Several remarks are due about Fig. (15). Mean data were used for spectral classes B0, B5, A0, .., M5, therefore this result is significant. The O-stars were omitted because of strong deviation from the relation. On Fig. (15), the sequence of spectral classes follows from upper right (B0) to lower left (M5) but the spectral classes run not in exactly the same order. These are details that are beyond the scope of this study. The primary significance of this result is that probably it is the same linear density relation, eq. (12), which holds for stars and for quasars. As stellar data could not be put in doubt, this result naturally corresponds to the result for quasars above, shown on Fig. (2). This is additional argument in favour of consistency. Besides, there is now tantalizing evidence that quasars and stars are somehow related. Could this link be expected in the process of their origin? This is yet another surprising hint which is worth to pursue further. One could ask also a question, what about the planets? Could they be fitted with the linear density eq (12)? Unfortunately, only the 9 big planets of the Solar system could be used for that presently and the result could not be significant. Yet, the hint also in this case may look promising. With reduced densities (to the same radius of $8.10^{13} \mathrm{~cm}$ ) of the 9 big planets, the coefficients in eq (12) are: $a=7 \cdot 10^{-11}$ and $b=0.2579$. Is this another tantalizing hint? 
Table 3. Summary of Data for the Solution of $\rho \sim=a+b \cdot r_{g r} / \mathbf{r}$

\begin{tabular}{|c|c|c|c|c|}
\hline & Coeff "a" & Coeff " $b$ " & Correlation Coeff & Range of the Reduced Density $\left[\mathbf{g} / \mathbf{c m}^{3}\right]$ to $\mathbf{r}=\mathbf{8 .} \mathbf{1 0}^{13}[\mathbf{c m}]$ \\
\hline \hline Eq (12) & 0 & 0.251549 & 1.0 & $0.02-0.25$ \\
\hline QSOs & 0.0002 & 0.251 & 0.998 & $0.8 .10^{-6}-2.3 .10^{-6}$ \\
\hline Stars B0-M5 & $-2.10^{-9}$ & 0.2505 & 0.998 & $1.1 .10^{-11}-1.10^{-8}$ \\
\hline 9 Planets & $7.10^{-11}$ & 0.258 & 0.998 & \\
\hline
\end{tabular}

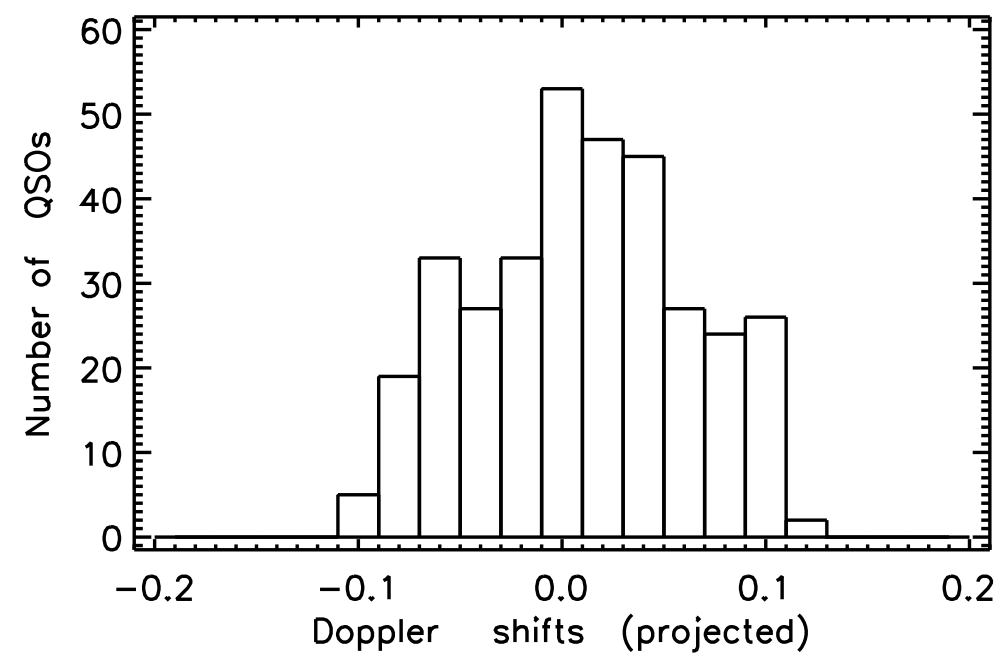

Fig. (16). Distribution of the projected Doppler shifts of the 341 sample quasars.

In Table 3, data for the solution of the linear density eq (12) is summarized for quasars, stars, and planets.

\section{THE DOPPLER-VELOCITIES DISTRIBUTION}

The distribution of the Doppler shifts of local quasars is yet unknown. It was discussed in [59] with a sample of 225 QSOs. There are several general assumptions, which seem realistic and are expected to be fulfilled:

- Doppler shifts reflect only the projections of ejection velocity along the line of sight. The real ejection velocities could be larger.

- Lower ejection velocities are more likely because of energy considerations. If the sample of QSOs is large enough, a peak in the distribution would therefore be expected around the zero Doppler shift.

- The distribution should have symmetry with respect to the zero velocity, if all directions of ejection have the same probability. There are reports [19], where the ejection of QSOs is noted to proceed along the minor (rotational) axis of the parent galaxy. This effect, if real, could introduce some distortion of the distribution with smaller samples of QSOs. With a large sample of galaxies and ejected quasars, however, these effects should cancel out and the distribution should be symmetric.

- Ejection velocities should be limited.

The distribution of the sample of 341 QSOs (225 from [59] and 116 from Table 2, present study) is shown on Fig. (16).
The distribution on Fig. (16) of projected Doppler shifts seems to correspond (more or less) to the above considerations. The highest projected velocity seems to be slightly over $30000 \mathrm{~km}$. s ${ }^{-1}$.

\section{DISCUSSION AND CONCLUDING REMARKS.}

Discussion about clustering of quasars around low redshift galaxies goes since about 40 years ago. The hypothesis of gravitational reddening makes it possible to get some of the physical characteristics of local quasars and to establish relations between them. These findings seem to be in agreement with the Arp's [45, 88] scenario - local quasars are probably ejected by active galactic nuclei due to yet unknown physical process. All conclusions and considerations here refer to only the sample of 341 QSOs (116 from this study, and 225 QSOs from [59]). Some conclusions seem quite radical. On the other hand, the sample presented is not negligible, and the conclusions are not unfounded. Generally, the results presented here confirm respective results from $[18,59]$.

Local quasars probably cluster around low redshift active galaxies, because probably being ejected by their respective nucleus. The highest projected velocity of ejection could be about $30000 \mathrm{~km}$. s ${ }^{-1}$. The physics of this ejection is yet unknown. Quasars are likely to be single objects, with dimensions close to their respective gravitational radius. A theory of such massive bodies does not yet exist. Their internal structure is yet unknown and our present knowledge seems insufficient to explain how such massive bodies exist. QSOs could evolve with decreasing density and redshifts, which could be due to disintegration of matter of yet unknown ori- 
gin and properties. At the beginning, the evolution of quasarredshifts due to decreasing density is very fast. This could explain the decreasing number of QSOs with $z>3$. Such quasars could evolve very fast to lower values of redshift. Another consequence to be expected by this evolution scenario is the increase of radius of quasars. The end product of evolution of local quasars could be small mass companion galaxies. Galaxies beget companion galaxies? This scenario should be considered seriously. If confirmed, such a scenario could probably require re-considering the theory of origin of galaxies in general. On the "density-redshift" diagram, there are no quasars below reduced density $\sim 0.02 \mathrm{~g} / \mathrm{cm}^{3}$. Could it be that QSOs have already evolved into galaxies? There could be yet another important implication. If quasars develop into galaxies, it would be possible to expect small gravitational components to exist also in the redshifts of some galaxies, which are still in transition (compact galaxies). Possible presence of gravitational reddening in distant galaxies may require introducing small corrections of the Hubble diagram and the Hubble relation.

It has been discussed in the past that if quasars would be of local origin that would contradict the Big Bang theory. This is not necessarily so. In our starting eq (1), taken from [60], a term of the expanding Universe is included. The expansion of the Universe and the gravitational reddening could be two different sources attributing to the redshifts of extragalactic sources. The problem would be how we could disentangle these two components? For local quasars, the observed redshifts could be decomposed rather easily to the three components - cosmological component, gravitational reddening, and a Doppler shift. Decomposition of these components in the redshifts of distant objects could prove to be much more difficult. It should be stressed, however, that the concept of local quasars does not contradict the cosmological expansion. It actually makes use of the expansion, when it comes to the effects of evolution. The gravitational redshift seems to be the largest component in local quasars and gravitational redshifts seem to be quantized, according to the Karlsson sequence. The negative result of searching for the Karlsson sequence, when using modern surveys does not necessarily mean that this sequence does not exist. The pattern of this sequence could be undetectable in modern surveys because of strong contribution by the two other components in the observed redshift, especially by the cosmological reddening. In the Arp's scenario [45, 88], the evolution of redshift proceeds in steps, each step corresponding to the next lower value of the Karlsson sequence. Quantized redshifts are, however, not compatible with our present physics and require new concepts. In order to obtain a sequence of specific gravitational redshifts, the gravitational potential of a quasar has to go by the quasar evolution through a sequence of specific (decreasing) values. How this should be possible is a mystery at present. Quasars are mysterious objects in other respects, too. We may need a deeper insight into the subatomic physics of matter to resolve these problems.

The procedure of determination of radii implies that QSOs should have a thermal outer layer, probably heated from below. This is in contradiction with the most popular theory of SQM. Larger gravitational redshifts seem to correspond to fainter quasars. As a result of the evolution, quasars' dimensions and luminosities seem to increase. Building stellar population around a quasar requires a mass. Therefore, QSOs should be losing mass in the process of evolution, in order to keep the total mass constant. There may be a hint that quasars' masses decrease for redshifts less than 0.60 . The process of evolution with decreasing density and building stars around a quasar reminds of the old hypothesis, suggested by Victor Ambartsumian. This concept has so far been largely neglected, because it was not possible to develop and to test specific models. In view of the recent developments this idea may get new attention. Some relations, shown in the section 5, appear to be in agreement with possible consequences of evolution due to disintegration: dimensions and luminosities increase with decreasing density. There is another possible hint, following from the "massdensity" relation. Quasars with larger masses could evolve more rapidly. Interesting evidence may come also from the "density - luminosity" relation. If confirmed, it would appear that more dense bodies are less luminous. Could this relation have a bearing on the much discussed problem of the "hidden" (dark) mass in the Universe?

A new finding here is the dependence of masses and luminosities of QSOs on distance. The evidence would appear to be that more distant quasars (to about $\mathrm{z}_{\mathrm{c}}=0.06$ ) are also more luminous and more massive. This trend is steep to about $\mathrm{z}_{\mathrm{gal}}=0.025$ and then seems to remain flat.

According to the disintegration scenario, in a group of quasars at about the same distance and having the same masses, the QSOs with largest redshifts should be most young.

Several relations for local quasars seem to exist, and each of them has similar (but not identical) counterpart relation for stars. For stars these relations are known for many years. Even more surprising is the linear density relation, eq (12). It appears that this equation holds for quasars, as well as for stars. Could it be that we may be dealing with a fundamental link, possibly having its roots in the origin of quasars and stars?

The possibility of having processes of disintegration as an alternative to the gravitational collapse as the origin of galaxies has been largely neglected in the past years. The implication of this alternative is not excluded, however, by the observational evidence for galaxies, and may be, even for stars. This could open enormous new possibilities.

\section{CONFLICT OF INTEREST}

The authors confirm that this article content has no conflicts of interest.

\section{ACKNOWLEDGEMENTS}

For this work, I used the SIMBAD and the Vizier databases, operated by CDS, Strasburg, France. My thanks are due to Dr D. Dimitrov, for his valuable help in preparation of the figures.

\section{REFERENCES}

[1] Antonucci, R. Unified models for active galactic nuclei and quasars. Astron Astrophys 1993; 31: 473-521.

[2] Djorgowski SG, Volonteri M, Springel V, Bromm V, Meylan G. The origins and the early evolution of quasars and supermassive black holes. Proceed. of XI Marcel Grossmann meeting on General Relativity. Singapore: World Scientific 2008; p. 340. 
[3] Kembhavi AK, Narlikar JV. Quasars and active galactic nuclei: an introduction. Cambridge: Cambridge University Press 1999.

[4] Lopez-Corredoira M. Pending problems in QSOs. IJAA 2011; 1(2): 73-82.

[5] Lopez-Corredoira M. Observational cosmology: caveats and open questions in the standard model. Recent Res Dev Astron Astrophys 2003; 1: 567-1.

[6] Greenstein JL, Schmidt M. The quasi-stellar radio sources 3C48 and 3C273. Astrophys J 1964; 140: 1-37.

[7] Hoyle F, Fowler WA. Gravitational red-shifts in quasi-stellar objects. Nature 1967; 213: 373-4.

[8] Das PK. Physical properties of collapsed objects with large central gravitational redshifts. Mon Not R Aston Soc 1976; 177: 391-408.

[9] Narlikar JV. Two astrophysical applications of conformal gravity. Ann Phys 1977; 107: 325-36.

[10] Narlikar JV, Arp HC. Flat spacetime cosmology - a unified framework for extragalactic redshifts. Astrophys J 1993; 405: 51-6.

[11] Canalizo G, Stockton A. 3C48: stellar populations and the kinematics of stars and gas in the host galaxy. Astrophys J 2000; 528: 20118 .

[12] Courbin F, Letawe G, Magain P, et al. On-axis spatially resolved spectroscopy of low redshift quasar host galaxies: HE1503+0228, at $\mathrm{z}=0.135$. Astron Astrophys 2002; 394: 863-72.

[13] Galianni P, Burbidge EM, Arp H, et al. The discovery of a high redshift $\mathrm{X}$ - ray emitting QSO very close to the nucleus of NGC7319. 2004 [Epub ahead of print].

[14] Karlsson KG. Possible discretization of quasar redshifts. Astron Astrophys 1971; 13: 333-5.

[15] Karlsson KG. On the existence of significant peaks in the quasar redshift distribution. Astron Astrophys 1977; 58: 237-40.

[16] Burbidge G, Napier WM. The distribution of redshifts in new samples of quasi-stellar objects. Astron J 2001; 121: 21-30.

[17] Arp H, Bi H, Chu Y, Zhu X. Periodicity of quasar redshifts. Astron Astrophys 1990; 239: 33-49.

[18] Panov KP. Study of possible local quasars I: the first sample. OAJ 2011; 4: 14-26.

[19] Arp HC. Catalogue of discordant redshift associations. Canada: APEIRON 2003.

[20] Arp HC. Quasars, redshifts, and controversies. Berkely, USA: Interstellar Medium 1987.

[21] Burbidge G. The reality of anomalous redshifts in the spectra of some QSOs and its implications. Astron Astrophys 1996; 309: 922.

[22] Burbidge GR. Noncosmological Redshifts. Publ Astron Soc Pac 2001; 113: 899-902.

[23] Hoyle F, Burbidge G. Anomalous redshifts in the spectra of extragalactic objects. Astron Astrophys 1996; 309: 335-44.

[24] Burbidge G, Hewitt A, Narlikar JV, Gupta PD. Associations between quasi-stellar objects and galaxies. Astrophys J Suppl Ser 1990; 74: 675-730.

[25] Williams LLR, Irwin M. Angular correlations between LBQS and APM: weak lensing by the large-scale structure. Mon Not R Aston Soc 1998; 298: 378-88.

[26] Bell MB. Further Evidence for Large Intrinsic Redshifts. Astrophys J 2002; 566: 705-11.

[27] Jain B, Scranton R, Sheth RK. Quasar-galaxy and galaxy-galaxy cross-correlations: model predictions with realistic galaxies. Mon Not R Aston Soc 2003; 345: 62-70.

[28] Lopez-Corredoira M, Gutierrez CM. Research on candidates for non-cosmological redshifts. USA: AIP Conf Proceed of First Crisis in Cosmology Conf 2006; vol. 822: pp. 75-96.

[29] Burbidge G, Napier WM. Associations of High-Redshift QuasiStellar Objects with Active, Low-Redshift Spiral Galaxies. Astrophys J 2009; 706: 657-64.

[30] Burbidge EM, Burbidge GR, Solomon PM, Strittmatter PA. Apparent associations between bright galaxies and quasi-stellar objects. Astrophys J 1971; 170: 233-40.

[31] Sulentic JW, Arp HC. The galaxy-quasar connection: NGC4319 and Markarian 205. I Direct imagery. Astrophys J 1987; 319: 68792.

[32] Carilli CL, van Gorkom JH, Stocke J. 3C232 and NGC 3067: quasars, galaxies, and absorption lines. Bull AAS 1988; 20: 1027.

[33] Carilli CL, van Gorkom JH, Stocke JT. Disturbed neutral hydrogen in the galaxy NGC3067 pointing to the quasar 3C232. Nature 1989; 338:134-6.
[34] Arp H. A QSO 2.4 arcsec from a dwarf galaxy - the rest of the story. Astron Astrophys 1999; 341: L5-8.

[35] Lopez-Corredoira M, Gutierrez CM. The field surrounding NGC7603: cosmological or non-cosmological redshifts? Astron Astrophys 2004; 421: 407-22.

[36] Lopez-Corredoira M, Gutierrez CM. First tentative detection of anisotropy in the QSO distribution around nearby edge-on spiral galaxies. Astron Astrophys 2007; 461: 59-69.

[37] Gosset E, Moreau O, Surdej J, et al. Surveys of ultraviolet-excess quasar candidates in large fields. Astron Astrophys Suppl Ser 1997; 123: 529-68.

[38] Arp H. X-ray Observations of NGC1097 and nearby quasars. Astrophys Space Sci 1998; 262: 337-61.

[39] Arp H, Burbidge EM, Chu Y, et al. NGC3628: Ejection activity associated with quasars. Astron Astrophys 2002; 391: 833-40.

[40] Arp H, Burbidge EM, Carosati D. Quasars and galaxy clusters paired across NGC4410. 2006 [Epub ahead of print].

[41] Burbidge EM, Burbidge G. QSOs in the field of the seyfert galaxy NGC5548. Publ Astron Soc Pac 2002; 114: 253-256.

[42] Burbidge EM. Spectra of two quasars possibly ejected from NGC 4258. Astron Astrophys 1995; 298: L1-4.

[43] Burbidge EM. Spectra of two x-ray emitting quasi-stellar objects apparently ejected from the Seyfert Galaxy NGC 2639. Astrophys J 1997; 484: L99-101.

[44] Burbidge EM, Burbidge G. Ejection of matter and energy from NGC 4258. Astrophys J 1997; 477: L13-5.

[45] Arp, H. Quasar creation and evolution into galaxies. J Astrophys Astron 1997; 18: 393-406.

[46] Arp H, Burbidge EM, Chu Y, et al. NGC 3628: ejection activity associated with quasars. Astron Astrophys 2002; 391: 833-40.

[47] Fan X, Narayanan VK, Lupton RH, et al. A survey of z $>5.8$ quasars in the sloan digital sky survey I: discovery of three new quasars and the spatial density of luminous quasars at $\mathrm{z} \sim 6$. Astron $\mathrm{J}$ 2001; 122: 2833-49.

[48] Simon LE, Hamann FW, Pettini M. Physical properties of absorbers in high redshift quasars. Rev Mex Astron Astrophys 2007; 29: 177.

[49] Maiolino R. Gas and dust in the most distant quasars. 2004; IAUS 222: 229-34.

[50] Vermeulen RC, Cohen MH. Superluminal motion statistics and cosmology. Astrophys J 1994; 430: 467-94.

[51] Zensus JA, Cohen MH, Unwin SC. The parsec-scale jet in quasar 3C345. Astrophys J 1995; 443: 35-53.

[52] Burbidge G. NGC 6212, 3C345, and other quasi-stellar objects associated with them. Astrophys J 2003; 586: L119-22.

[53] Bahcall JN, Kirhakos S, Saxe DH. Hubble space telescope images of a sample of 20 nearby luminous quasars. Astrophys J 1997; 479: 642-58.

[54] McLure RJ, Kukula MJ, Dunlop JS, et al. A comparative HST imaging study of the host galaxies of radio-quiet quasars, radioloud-quasars, and radio galaxies-I. Mon Not R Aston Soc 1999; 308: 377-404.

[55] Di Matteo T, Springel V, Hernquist L. Energy input from quasars regulates the growth and activity of black holes and their host galaxies. Nature 2004; 433: 604-7.

[56] Dunlop JS, McLure RJ, Kukula MJ, et al. Quasars, their host galaxies, and their central black holes. Mon Not R Aston Soc 2003; 340 : 1095-135.

[57] Grogin NA, Conselice CJ, Chatzichristou E, et al. AGN host galaxies at $\mathrm{z} \sim 0.4-1.3$ : Bulge - dominated and lacking merger $-\mathrm{AGN}$ connection. Astrophys J 2005; 627: L97-100.

[58] Cattaneo A, Faber SM, Binney J, et al. The role of black holes in galaxy formation and evolution. Nature 2009; 460: 213-9.

[59] Panov KP, Dimitrov DP. Study of possible local quasars II. A sample of 255 QSOs. Volume of contributions dedicated to Prof M. Contadakis, Thessaloniki, (in print).

[60] Burbidge G. Two universes. Astrophys Sp Sci 1996; 244: 169-76.

[61] Veron-Cetty MP, Veron P. A catalogue of quasars and active nuclei. $13^{\text {th }}$ ed. Astron Astrophys 2010; 518A: A10.

[62] Croom SM, Smith RJ, Boyle BJ, et al. The 2dF QSO Redshift Survey $-\mathrm{V}$. The 10k catalogue. Mon Not R Aston Soc 2001; 322: L29-36.

[63] Arp H, Surdej J, Swings J-P. Two quasars seen near the spiral galaxy NGC 470. Astron Astrophys 1984; 138:179-82.

[64] Arp H, Duhalde O. Quasars near NGC 520. Publ Astron Soc Pac 1985; 97: 1149-57. 
[65] Arp H. Quasars associated with NGC 613, NGC 936 and NGC 941. Astrophys Space Sci 2006; 301:117-26.

[66] Arp H, Roscoe D, Fulton C. Periodicities of quasar redshifts in large area surveys. NY: BKS Press 2005.

[67] Abazajian K, Adelman-McCarthy JK, et al. The second data release of the sloan digital sky survey. Astron J 2004; 128: 502-12.

[68] Burbidge EM. A group of quasi-stellar objects closely associated with NGC 1068. Astrophys J 1999; 511: L9-L11.

[69] Arp H, Sulentic JW. Three quasars near the spiral arms of NGC 1073. Astrophys J 1979; 229: 496-502.

[70] Kaaret P. Optical Sources near the bright X-Ray Source in NGC 1073. Astrophys J 2005; 629: 233-8.

[71] Arp H. Pairs of X-ray sources across Seyferts: the NGC 4235 field. Astron Astrophys 1997; 328: L17-L20.

[72] Arp H. Further Observations and Analysis of Quasars near Companion Galaxies. Astrophys J 1983; 271: 479-506.

[73] Arp H. Quasars near companion galaxies. Astrophys J 1981; 250: 31-42.

[74] Arp H. High - redshift objects near the companion galaxies to NGC 2859. Astrophys J 1980; 240: 415-20.

[75] Burbidge EM, Burbidge G, Arp HC, Zibetti S. QSOs associated with M82. Astrophys J 2003; 591: 690-4.

[76] Burbidge EM, Burbidge G, Arp HC, Napier WM. An anomalous concentration of QSOs around NGC 3079. Astrophysics 2005 [Epub ahead of print].
[77] Arp H, Sulentic JW, di Tullio G. Quasars aligned across NGC 3384. Astrophys J 1979; 229: 489-95.

[78] Chu Y, Wei J, Hu J, et al. Quasars around the Seyfert Galaxy NGC 3516. Astrophys J 1998; 500: 596-8.

[79] Arp H. Two newly discovered quasars closely spaced across a galaxy. Astrophys J 1984; 283: 59-63.

[80] Arp H, Gavazzi G. A third quasar close to NGC 3842. Astron Astrophys 1984; 139: 240-2.

[81] Zhu X, Chu Y, Wei J, et al. A quasar possibly ejected from NGC 4579. Chin Sci Bull 2000; 45: 886-8.

[82] Arp H, Russell D. A possible relationship between quasars and clusters of galaxies. Astrophys J 2001; 549: 802-19.

[83] Arp HC, Burbidge EM, Chu Y, Zhu X. X-Ray emitting QSOs ejected from Arp 220. Astrophys J 2001; 553: L11-3.

[84] Arp H. The surroundings of disturbed, active galaxies. Astrophys J 2001; 549: 780-801.

[85] Weedman DW. A photometric study of markarian galaxies. Astrophys J 1973; 183: 29-39.

[86] Arp H, Fulton C. A cluster of high redshift quasars with apparent diameter 2.3 degrees. Arix 2008 [Epub ahead of print].

[87] Ambartsumian VA. La structure et l'evolution de l'univers. $11^{\text {th }} \mathrm{ed}$. Bruxelles: Conseil de Physic Solvay 1958.

[88] Arp H. Evolution of quasars into galaxies and its implications for the birth and evolution of matter. APEIRON 1998; 5: 135-42.

Received: February 18, 2013

Revised: May 08, 2013

Accepted: May 08, 2013

(C) Kiril P. Panov; Licensee Bentham Open.

This is an open access article licensed under the terms of the Creative Commons Attribution Non-Commercial License (http://creativecommons.org/licenses/by-nc/3.0/) which permits unrestricted, non-commercial use, distribution and reproduction in any medium, provided the work is properly cited. 